\title{
Evaluating snail-trail frameworks for leader-follower behavior with agent-based modeling
}

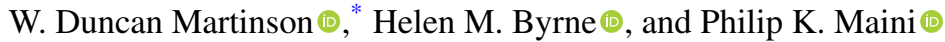 \\ Mathematical Institute, University of Oxford, Woodstock Road, Oxford OX2 6GG, United Kingdom
}

(Received 30 June 2020; revised 1 October 2020; accepted 18 November 2020; published 14 December 2020)

\begin{abstract}
Branched networks constitute a ubiquitous structure in biology, arising in plants, lungs, and the circulatory system; however, the mechanisms behind their creation are not well understood. A commonly used model for network morphogenesis proposes that sprouts develop through interactions between leader (tip) cells and follower (stalk) cells. In this description, tip cells emerge from existing structures, travel up chemoattractant gradients, and form new networks by guiding the movement of stalk cells. Such dynamics have been mathematically represented by continuum "snail-trail" models in which the tip cell flux contributes to the stalk cell proliferation rate. Although snail-trail models constitute a classical depiction of leader-follower behavior, their accuracy has yet to be evaluated in a rigorous quantitative setting. Here, we extend the snail-trail modeling framework to two spatial dimensions by introducing a novel multiplicative factor to the stalk cell rate equation, which corrects for neglected network creation in directions other than that of the migrating front. Our derivation of this factor demonstrates that snail-trail models are valid descriptions of cell dynamics when chemotaxis dominates cell movement. We confirm that our snail-trail model accurately predicts the dynamics of tip and stalk cells in an existing agent-based model (ABM) for network formation [Pillay et al., Phys. Rev. E 95, 012410 (2017)]. We also derive conditions for which it is appropriate to use a reduced, one-dimensional snail-trail model to analyze ABM results. Our analysis identifies key metrics for cell migration that may be used to anticipate when simple snail-trail models will accurately describe experimentally observed cell dynamics in network formation.
\end{abstract}

DOI: 10.1103/PhysRevE.102.062417

\section{INTRODUCTION}

Branching networks are a near-omnipresent biological structure, appearing in plant roots [1,2], lungs [3,4], kidneys $[5,6]$, and the circulatory network $[7-11]$. The geometry of such patterns highlights their biological importance, as branched networks improve the efficiency of nutrient transport by maximizing the surface area across which molecules are exchanged [12]. Furthermore, changes in network morphology may lead to, or are a sign of, severe disruptions in healthy tissues and organs. In angiogenesis (the formation of new blood vessels from pre-existing ones), for instance, pathologic network formation has been associated with blindness, retinopathy, and delayed wound healing; additionally, it is stimulated to occur by cancerous tumors to supply nutrients for their sustained growth [9,13-19]. Due to the widespread nature of branching networks and their crucial importance in maintaining homeostasis, increasing research has been devoted to understanding the molecular, physical, and mechanical factors behind their morphogenesis [8,12]. Complete knowledge of this process, however, remains elusive because of its inherent multiscale nature: although network formation is most readily observed at the level of tissue, the growth of new sprouts depends on the collective migration of cells, which are themselves regulated by molecules and genes.

One model for network morphogenesis in general systems proposes that sprout formation is guided by two cell types:

\footnotetext{
*william.martinson@maths.ox.ac.uk
}

leader (tip) cells and follower (stalk) cells. In this description, external chemical and/or mechanical signals cause tip cells to move from existing structures; they also guide the direction in which tip cells migrate. Stalk cells, meanwhile, provide structural support for the new network and are assumed to proliferate along the path of tip cells. In angiogenesis, for example, tip cells are known to migrate toward increasing concentrations of vascular endothelial growth factor (VEGF) and other known chemoattractants that are generically called tumor angiogenic factors (TAFs) [7,9,17,19-21], while stalk cells form a lumen in the new network through which blood can flow [7,17,22-25].

Leader-follower models for network morphogenesis also include mechanisms by which new branches and loops form. New sprouts, for instance, can be initiated when tip cells divide or when new tip cells emerge along the growing network, while closed loops are created when a tip cell merges with another tip or stalk cell (a process called anastomosis).

Many mathematical representations of leader-follower models have been proposed to describe network morphogenesis at various levels of detail [26-29]. Discrete models, for instance, track the progression of individual cells and can provide insight into in vitro and in vivo observations of the resulting network geometry. Such discrete models may also be coupled with continuum differential equations for quantities at the subcellular scale to create so-called "hybrid" models [27-30]. Individual-based modeling approaches can either be on-lattice, in which case cell positions are restricted to certain spatial locations [31-38], or off-lattice, where cell dynamics are described by stochastic differential equations 
[39-42]. The development and simulation of discrete models is aided by specialized software, such as Microvessel Chaste and CompuCell3D (to name but two), which support many of the approaches listed above and efficiently generate solutions $[43,44]$.

Other mathematical models for leader-follower behavior treat tip and stalk cell densities as continuous variables and track their evolution in space and/or time via differential equations [45-49]. Although many such continuum models for angiogenesis describe the evolution of spatially averaged populations (and, hence, cannot resolve individual network structure), they are still useful as they tend to be more amenable to mathematical analysis.

A broad class of continuum leader-follower models phenomenologically assumes that the rate of sprout creation increases proportionally to the net tip cell flux (this represents the "snail-trail" assumption that stalk cells proliferate along the path of moving tip cells). Such snail-trail models for angiogenesis were originally proposed to model branching patterns in fungal growth [50,51], but have since been extended to explain network morphogenesis from in vivo assays of angiogenesis [46-48,52] and plant roots [53].

The earliest snail-trail models were formulated in one spatial dimension (1D), but have since been constructed in two spatial dimensions (2D) to examine the spatial variation of cell densities in arbitrary geometries [54,55]. Since the net tip cell flux becomes a vector quantity in higher dimensions, a common modeling choice in $2 \mathrm{D}$ is to use the magnitude of this vector to calculate the sprout density rate of change $[33,35]$. In other words, if $\mathbf{J}$ denotes the net tip cell flux vector, then the rate of increase in network density, $\rho(\mathbf{x}, t)$, is

$$
\frac{\partial \rho}{\partial t}=\|\mathbf{J}\|_{2}=\sqrt{\mathbf{J} \cdot \mathbf{J}}=\sqrt{\left(J_{x}\right)^{2}+\left(J_{y}\right)^{2}},
$$

where $\|\cdot\|_{2}$ denotes the $L_{2}$ norm, and $J_{x}$ and $J_{y}$ denote the $x$ and $y$ components of the net tip cell flux vector, respectively.

Although snail-trail models represent a simple and analytically tractable approach for describing tip and stalk cell dynamics during network formation, they rely on phenomenological arguments for their construction. As a result, the conditions under which snail-trail models will accurately describe experimentally observed cell dynamics remain unclear. To our knowledge, the validation of snail-trail models has largely focused on achieving qualitative agreement with experiments; there has been little work to evaluate snail-trail models in a more rigorous theoretical setting. Additionally, many snail-trail models have investigated vessel growth in 1D, but it remains unclear when such spatially reduced systems are good approximations to $2 \mathrm{D}$ results.

\section{A. Article outline}

In this article, we develop a 2D snail-trail model for tip and stalk cell dynamics and extend its underlying framework by introducing a novel multiplicative factor in the differential equation describing sprout density. We propose that this multiplicative factor must be included to correct Eq. (1) because the tip cell net flux term neglects sprout formation in directions other than that of the moving solution front. We derive a formula for the multiplicative factor, which suggests that snail-trail models are valid approximations of tip and stalk cell dynamics when movement is dominated by chemotaxis. Hence, we identify some of the assumptions that are implicitly invoked when snail-trail models are used to analyze network formation. We confirm the accuracy of our formula by comparing numerical solutions of the $2 \mathrm{D}$ snail-trail model with ensemble averages of an existing agent-based model (ABM) for network formation [32]. We conclude with a discussion of when our 2D snail-trail model can be reduced to a 1D system, and identify conditions for which it is appropriate to use 1D snail-trail models to analyze experimental data.

\section{SNAIL-TRAIL MODEL DEVELOPMENT IN 2D}

Our 2D snail-trail model tracks the densities of tip and stalk cells within the unit square $(x, y) \in[0,1] \times[0,1]$ and time interval $t \in\left[t_{0}, \infty\right)$, where $t_{0} \geqslant 0$ (all quantities have been nondimensionalized). We assume that there are sufficient cell numbers to justify a continuous approximation, such that in Cartesian coordinates we may use continuous functions $n(x, y, t)$ and $e(x, y, t)$ to describe the tip and stalk cell densities, respectively. Additionally, we assume that the system contains a generic chemoattractant (a TAF) whose concentration is denoted by $c(x, y, t)$. In general, the value of $c$ is governed by a differential equation that may include terms corresponding to diffusion and/or uptake by tip and stalk cells [46,56,57]. However, in this article we are most interested in determining appropriate equations for describing cell movement and proliferation. For this reason, we simplify our model by assuming that the TAF field is at steady state, such that it is independent of $t$ and hence may be written as a function $c(x, y)$.

Our basis for modeling the tip cell density is the continuity equation

$$
\underbrace{\frac{\partial n}{\partial t}}_{\text {tip cell rate of change }}+\underbrace{\nabla \cdot \mathbf{J}}_{\text {net movement of tip cells }}=\underbrace{F(n, e, c),}_{\text {tip cell production/elimination }}
$$

where $\mathbf{J}$ is the net tip cell flux vector and the function $F$ accounts for tip cell production and elimination. Following previous assumptions in snail-trail models [46,47,54,55], we consider cell movement as a biased random walk, in which the bias is toward increasing TAF gradients; this simplistic method of accounting for such types of motion is common within the modeling community $[39,40,58,59]$. Thus, the net flux of tip cells is given by

$$
\mathbf{J}=\underbrace{-D \nabla n}_{\text {random motion }}+\underbrace{\chi(c) n \nabla c}_{\text {chemotaxis }}
$$

where $\nabla$ is the gradient operator, $D$ is a positive random motion coefficient, and $\chi(c)$ is a positive function that measures the chemotactic sensitivity of cells to the TAF. We assume for simplicity that $\chi(c)=\chi$ is constant, although it is possible to use other functional forms that account for cell desensitization to TAF in high concentrations (for examples of such functions, we refer to Ref. [60]). 
We represent the creation of new sprouts by an increase of tip cells. Observations of network morphogenesis in several angiogenesis assays indicate that sprout branching depends on the TAF concentration [61-64], thus we assume that tip cells are produced at a rate proportional to the TAF concentration. The particular form of the branching term we employ was originally developed in a previous snail-trail model for angiogenesis by Byrne and Chaplain [47], who used it to explain in vivo observations such as the brush-border effect [65]. We consider two dominant sources of tip cell elimination: tip-to-tip and tip-to-sprout anastomosis, and assume that these processes occur at rates proportional to the product of the relevant cell densities. This means that the net rate of tip cell production is given by

$$
F(n, e, c)=\underbrace{\lambda n c}_{\text {branching }}-\underbrace{\beta_{e} n e}_{\text {tip-to-sprout anastomosis }}-\underbrace{\beta_{n} n^{2}}_{\text {tip-to-tip anastomosis }},
$$

where $\lambda, \beta_{e}$, and $\beta_{n}$ are positive parameters.

In accordance with the snail-trail framework, we consider the rate of blood vessel production to be proportional to the magnitude of the net tip cell flux. We thus follow the formulation given by Eq. (1). However, since we are interested in cell, rather than sprout, density, we divide this rate by $h$, a constant that represents the length of a typical stalk cell. Additionally, in the stalk cell density rate equation we introduce a corrective factor $\kappa(x, y)$, which is new to the 2D snail-trail framework (motivation for this factor is found below). The complete nondimensional system is therefore given by

$$
\begin{gathered}
\frac{\partial n}{\partial t}=D \nabla^{2} n-\chi \nabla \cdot(n \nabla c)+\lambda n c-\beta_{e} n e-\beta_{n} n^{2}, \\
\frac{\partial e}{\partial t}=\frac{\kappa(x, y)}{h}\|\chi n \nabla c-D \nabla n\|_{2} .
\end{gathered}
$$

The snail-trail model given by Eqs. (3) and (4) is closed with no-flux boundary conditions

$$
(\chi n \nabla c-D \nabla n) \cdot \mathbf{a}=0,
$$

where $\mathbf{a}$ is the outward unit normal vector, and initial conditions

$$
n\left(x, y, t_{0}\right)=G(x, y), \quad e\left(x, y, t_{0}\right)=H(x, y),
$$

where $G(x, y)$ and $H(x, y)$ are nonnegative functions. Boundary conditions are not required for the stalk cell density because its rate equation is a first-order ordinary differential equation (ODE) with respect to time $t$. The TAF concentration $c(x, y)$ is prescribed in simulations, since its value is independent of time.

The multiplicative factor $\kappa(x, y)$ has been included in Eq. (4) because the net tip cell flux term, used to calculate the stalk cell density rate of change, neglects sprout growth in directions other than that of the invading front. To see this, we may imagine decomposing the $2 \mathrm{D}$ tip cell density into four subpopulations: $n_{x^{+}}, n_{x^{-}}, n_{y^{+}}, n_{y^{-}}$, each corresponding to the density of tip cells moving in the positive $x$ direction within a single time step, the negative $x$ direction within a single time step, and so on. Figure 1(a) presents a sketch of these quantities for a given point. New sprouts (or, equivalently, vessels) will develop along each of these directions, and will therefore contribute to the total vessel density created within a given time step. The net tip cell flux term, however, ignores some of these contributions by calculating the net vessel

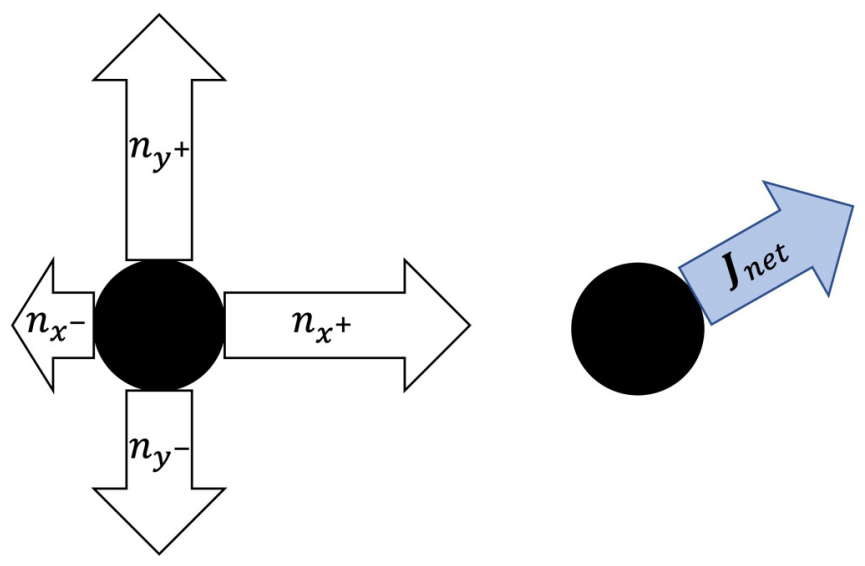

FIG. 1. The net flux vector $\mathbf{J}_{\text {net }}$ underestimates the total sprout/vessel density rate of change. (a) From a given point (black circle), one may measure the total flux of tip cells moving in any cardinal direction; each of these quantities should be considered in snail-trail models when calculating the vessel density proliferation rate. (b) The vector $\mathbf{J}_{\text {net }}$, however, only considers the flow of cells in the resulting net direction, which is always less than or equal to the total quantity of material that moves within a given time period.

density produced in a direction defined by $\left(n_{x^{+}}-n_{x^{-}}\right)$and $\left(n_{y^{+}}-n_{y^{-}}\right)$, which is represented in Fig. 1(b). As a result, the magnitude of the net tip cell flux vector tends to underestimate the total vessel production rate, unless a corrective factor is included.

While this multiplicative factor is new to 2D snail-trail models, similar corrections have been introduced in 1D systems of this type $[32,46]$. However, the authors in those studies used an ad hoc approach to determine its value, which was assumed to be constant. In the next section, we present a general argument for determining how $\kappa$ varies with spatial position. We demonstrate in Sec. V that our method for determining $\kappa$ results in the $2 \mathrm{D}$ snail-trail model being able to approximate the ensemble average behavior of an ABM for angiogenesis better than other approaches.

We remark that $\kappa$ corrects for underestimated vessel production from the net tip cell flux, not from other activities such as branching or anastomosis. Hence, our derivation of $\kappa$ will not depend on branching or anastomosis, and so we neglect these processes in Sec. III.

\section{DERIVATION OF A FUNCTIONAL FORM FOR THE SNAIL-TRAIL MULTIPLICATIVE FACTOR}

We have argued that $\kappa$ corrects for neglected vessel production in directions other than that of the migrating front. Thus, if $\mathbf{J}_{\text {net }}$ measures the average net tip cell flux within a time step $\Delta t$ and spatial interval $h$, then $\kappa\left\|\mathbf{J}_{\text {net }}\right\|_{2}$ is equal to the (true) average vessel density that is produced within this interval (note that we have absorbed any dependence of $h$ and $\Delta t$ into $\kappa$ ). To derive a function for $\kappa$, we examine how the above statement translates into a discrete setting.

We consider a general on-lattice framework to represent the discrete movement of tip and stalk cells following a snail-trail assumption. In this ABM, we describe tip cell movement as a biased random walk toward increasing concentrations of TAF. 
The ABM is assumed to have $N$ lattice sites that are equally spaced with step size $h$, where $h$ has the same interpretation as in Eq. (4) (i.e., it represents the length of a typical cell). We assume, for sake of simplicity, that a tip cell length is equivalent to that of a stalk cell; at the end of this section we discuss how the following results change when this assumption is relaxed. Just as for the continuous snail-trail model, we assume that the TAF concentration $c(x, y)$ is prescribed and is independent of time $t$.

Within a time step $\Delta t$, tip cells are chosen to move with a constant probability $P_{m}$. Once chosen to move, the direction in which the tip cell travels is selected according to the following probabilities: if we denote the probability of moving left as $P_{x^{-}}$, right as $P_{x^{+}}$, up as $P_{y^{+}}$, and down as $P_{y^{-}}$, then these values are defined at $\left(x_{i}, y_{j}\right) \in(0,1)^{2}$ as

$$
\begin{aligned}
& P_{x^{ \pm}}=\frac{1 \pm g_{x}\left(x_{i}, y_{j}\right)}{4}, \\
& P_{y^{ \pm}}=\frac{1 \pm g_{y}\left(x_{i}, y_{j}\right)}{4},
\end{aligned}
$$

where for $0<i, j<(N-1)$,

$$
\begin{aligned}
& g_{x}\left(x_{i}, y_{j}\right):=k\left[c\left(x_{i}+h, y_{j}\right)-c\left(x_{i}-h, y_{j}\right)\right], \\
& g_{y}\left(x_{i}, y_{j}\right):=k\left[c\left(x_{i}, y_{j}+h\right)-c\left(x_{i}, y_{j}-h\right)\right] .
\end{aligned}
$$

For example, if $g_{x}=1 / 2$ and $g_{y}=-1 / 4$, then $P_{x^{-}}=1 / 8$, $P_{x^{+}}=3 / 8, P_{y^{+}}=3 / 16$, and $P_{y^{-}}=5 / 16$ (i.e., the tip cell is more likely to move in the right and downward directions). It can be readily verified that the four probabilities in Eq. (7) sum to 1 . The parameter value $k$ in Eq. (8) ensures no numerator in Eq. (7) can become negative (so that $\left|g_{x}(x, y)\right| \leqslant 1$ and $\left.\left|g_{y}(x, y)\right| \leqslant 1\right)$; the value of $k$ does not vary with respect to location.

We incorporate a discrete version of the snail-trail assumption by having stalk cells proliferate in the space left empty by a moving tip cell. In other words, any new vessel production within a time step $\Delta t$ is equal to the number of times tip cells move, since stalk cells will proliferate to occupy the resulting empty space.

This leads us to immediately identify a discrete analogue of $\left\|\mathbf{J}_{\text {net }}\right\|_{2}$ : it is simply the expected net number of jumps that tip cells make from lattice site $\left(x_{i}, y_{j}\right)$ in the $x$ and $y$ directions (we use expected values here because of the stochastic nature of the discrete model). We may then interpret the value of $\kappa$ as the total amount of new vessel density produced for every unit of net tip cell movement.

We now use the rules of the $\mathrm{ABM}$ to compute the expected number of jumps that tip cells make in any direction. We define $X_{R}, X_{L}, Y_{U}, Y_{D}$ to be random variables that, respectively, measure the total number of rightward/leftward/upward/downward jumps that originate from lattice point $\left(x_{i}, y_{j}\right)$. Since cells may only move in one of these four directions, we model these random variables using multinomial distributions. We also define the random variables $X_{\text {net }}=X_{R}-X_{L}$ and $Y_{\text {net }}=Y_{U}-Y_{D}$, which measure the net number of rightward/upward jumps made at lattice site $\left(x_{i}, y_{j}\right)$, respectively. By the argument above, the direction of the net tip cell flux vector is defined by $J_{x}:=\mathbb{E}\left[X_{\text {net }}\right]$ and $J_{y}:=$ $\mathbb{E}\left[Y_{\text {net }}\right]$. Its magnitude, $\left\|\mathbf{J}_{\text {net }}\right\|_{2}$, is given as $\sqrt{\left(J_{x}\right)^{2}+\left(J_{y}\right)^{2}}$.
Since we have reasoned that $\kappa\left\|\mathbf{J}_{\text {net }}\right\|_{2}$ is the total vessel density produced (or, equivalently, total jumps that occur) from a given lattice site within the time step $\Delta t$ and spatial interval $h$, it follows that when the value of $\left\|\mathbf{J}_{\text {net }}\right\|_{2}$ is normalized to $1, \kappa$ becomes equal to the total number of jumps from lattice site $\left(x_{i}, y_{j}\right)$. We exploit this information to calculate the expected values of our random variables: for example, the probability of executing $m$ rightward jumps becomes

$$
P\left(X_{R}=m\right)=\left(\begin{array}{c}
\kappa \\
m
\end{array}\right)\left(P_{x^{+}}\right)^{m}\left(1-P_{x^{+}}\right)^{\kappa-m},
$$

where $\left(\begin{array}{l}\kappa \\ m\end{array}\right)$ is the binomial coefficient. Similar equations hold for $X_{L}, Y_{U}$, and $Y_{D}$. The expected values of $X_{R}, X_{L}, Y_{U}$, and $Y_{D}$ are thus given by

$$
\begin{array}{ll}
\mathbb{E}\left[X_{R}\right]=\kappa P_{x^{+}}, & \mathbb{E}\left[X_{L}\right]=\kappa P_{x^{-}}, \\
\mathbb{E}\left[Y_{U}\right]=\kappa P_{y^{+}}, & \mathbb{E}\left[Y_{D}\right]=\kappa P_{y^{-}},
\end{array}
$$

when $\left\|\mathbf{J}_{\text {net }}\right\|_{2}$ is normalized to 1 , so that

$$
\begin{aligned}
& \mathbb{E}\left[X_{\text {net }}\right]=\mathbb{E}\left[X_{R}\right]-\mathbb{E}\left[X_{L}\right]=\kappa\left(P_{x^{+}}-P_{x^{-}}\right), \\
& \mathbb{E}\left[Y_{\text {net }}\right]=\mathbb{E}\left[Y_{U}\right]-\mathbb{E}\left[Y_{D}\right]=\kappa\left(P_{y^{+}}-P_{y^{-}}\right) .
\end{aligned}
$$

Since Eqs. (9) and (10) are applicable when $\left\|\mathbf{J}_{\text {net }}\right\|_{2}=1$, we use Eq. (7) to deduce that

$$
\begin{aligned}
\left\|\mathbf{J}_{\text {net }}\right\|_{2} & =\sqrt{\left(J_{x}\right)^{2}+\left(J_{y}\right)^{2}} \\
& =\sqrt{\mathbb{E}\left[X_{\text {net }}\right]^{2}+\mathbb{E}\left[Y_{\text {net }}\right]^{2}} \\
& =\frac{\kappa}{2} \sqrt{\left(g_{x}\right)^{2}+\left(g_{y}\right)^{2}}=1,
\end{aligned}
$$

with $g_{x}$ and $g_{y}$ defined by Eq. (8). Hence,

$$
\kappa=\frac{2}{\sqrt{\left(g_{x}\right)^{2}+\left(g_{y}\right)^{2}}} .
$$

From this equation, it is clear that $\kappa$ is nonnegative.

We may apply Taylor's theorem to simplify Eq. (11): assuming that the average cell length is sufficiently small so that $0<h \ll 1$, we may write $g_{x}$ as

$$
\begin{aligned}
g_{x}(x, y) & =k[c(x+h, y)-c(x-h, y)] \\
& =k\left[2 h \frac{\partial c}{\partial x}+O\left(h^{3}\right)\right] \\
& \approx 2 k h \frac{\partial c}{\partial x}(x, y) .
\end{aligned}
$$

A similar formula holds for $g_{y}$. Substitution of these expressions into Eq. (11) yields

$$
\kappa(x, y) \approx \frac{1}{k h\|\nabla c(x, y)\|_{2}},
$$

hence $\kappa(x, y)$ is inversely proportional to the magnitude of the local TAF gradient. We remark that $\kappa$ is a function of space because the TAF gradient may depend on the variables $x$ and $y$. Although we do not consider cases in which the TAF field is time-dependent, it is straightforward to extend our analysis to such cases.

It is possible to further transform Eq. (12) so that $\kappa(x, y)$ is in terms of the continuum parameters $D$ and $\chi$. This 
simplification follows from the relationship between biased random walk models and advection-diffusion equations $[32,66]$. Namely, if $P_{m}$ denotes the probability that a tip cell moves within a time step $\Delta t$, then the relationship between the discrete and continuum parameters is

$$
D=\lim _{h \rightarrow 0, \Delta t \rightarrow 0} \frac{P_{m} h^{2}}{4 \Delta t}, \quad \chi=\lim _{h \rightarrow 0, \Delta t \rightarrow 0} \frac{P_{m} k h^{2}}{\Delta t},
$$

under the assumption that the above limits exist, are nonzero, and are finite. Substituting Eq. (13) into Eq. (12) yields

$$
\kappa(x, y)=\frac{4 D}{h \chi\|\nabla c(x, y)\|_{2}}=\frac{\mu h}{\chi\|\nabla c(x, y)\|_{2}},
$$

where we have defined $\mu:=P_{m} / \Delta t$.

Equation (14) predicts that $\kappa \rightarrow \infty$ when $\chi\|\nabla c\|_{2} \rightarrow 0$. Since both $\chi$ and $\nabla c$ appear in the snail-trail model as terms describing the directed movement of tip cells due to chemotaxis, it follows that our equation for $\kappa(x, y)$ is not applicable when chemotaxis is not the dominant form of motion for tip cells. This breakdown in our analysis occurs because $\kappa$ is attempting to correct for a near-zero stalk cell production rate that would occur in its absence. When there is no chemotaxis in the snail-trail model, tip cells move randomly with no well-defined direction and $\left\|\mathbf{J}_{\text {net }}\right\|_{2} \approx 0$. If we ignore the contribution of $\kappa$, then Eq. (1) predicts that there would be almost no vessel production. However, in the ABM we find that when tip cells move randomly, they travel a large number of short distances on average but still produce a nonzero number of stalk cells. Thus, $\kappa$ must take on large values to ensure that the continuum model yields a nonzero vessel production rate and matches the ABM results.

We therefore view Eq. (14) as a "leading-order" approximation for $\kappa$ that is accurate when $\|\nabla c\|_{2}$ is sufficiently large, so that chemotaxis dominates tip cell movement.

We conclude this section by discussing how our analysis is affected when tip cells and stalk cells do not have the same length $h$. Indeed, in certain biologically relevant scenarios tip cells can extend filopodia and, as a result, may become more elongated than stalk cells [62]. We can account for different cell lengths in our modeling framework by defining the length of the ABM lattice to be equal to that of a tip cell $\left(h_{\mathrm{TC}}\right)$, and denoting the length of a stalk cell as the separate quantity $h_{\mathrm{EC}}$. Since we have only employed the ABM lattice length to derive Eq. (14), our expression for $\kappa(x, y)$ is unchanged (the value of $h$ in that equation, however, should be written as $h_{\mathrm{TC}}$ for clarity). Although this result appears counter-intuitive, we note that by defining an ABM lattice length as $h_{\mathrm{TC}}$, we have also made the assumption that the length of a new vessel is equal to $h_{\mathrm{TC}}$. Thus, we would expect the vessel density rate of change to be unaffected when the tip and stalk cell lengths are different.

The stalk cell density rate of change given by Eq. (4), however, changes when $h_{\mathrm{TC}} \neq h_{\mathrm{EC}}$. To see this, we may imagine a scenario in which a tip cell is as long as $\gamma$ stalk cells, such that $h_{\mathrm{TC}}=\gamma h_{\mathrm{EC}}$. Since we determined above that $\kappa(x, y)=$ $\mu h_{\mathrm{TC}} /\left(\chi\|\nabla c\|_{2}\right)$, this means that the quantity $\kappa(x, y) / h_{\mathrm{EC}}$ that appears in Eq. (4) reduces to $\mu \gamma /\left(\chi\|\nabla c\|_{2}\right)$. Hence, the stalk cell density rate of change is multiplied by $\gamma$ when tip and stalk cells are of different lengths. This result makes sense because changing the stalk cell length should only affect the density of stalk cells (not sprouts) that proliferate along the path of moving tip cells.

\section{MULTIPLICATIVE FACTORS ARE NECESSARY IN THE 2D SNAIL-TRAIL STALK CELL EQUATION}

In this section, we confirm that the multiplicative factor $\kappa(x, y)$ must be included in the 2D snail-trail model by comparing its numerical solutions to those of a 2D ABM for angiogenesis previously developed by Pillay et al. [32]. We use this particular ABM (henceforth denoted as the $\mathrm{P}-\mathrm{ABM}$ ) because it is a discrete, cell-based representation of the verbal description that the classical snail-trail formulation purports to model. In other words, tip and stalk cell movement in the $\mathrm{P}-\mathrm{ABM}$ follow the same rules as those in the discrete model presented in Sec. III; the P-ABM is a more detailed description of network formation, however, because it directly models phenomena such as sprout branching and anastomosis that were ignored in the previous section. Full details on the $\mathrm{P}-\mathrm{ABM}$ are found in Appendix A.

Figure 2 presents the ensemble average of $1000 \mathrm{P}-\mathrm{ABM}$ tip and stalk cell solutions subject to the TAF field $c(x, y)=x$ at two time points, using the initial conditions and parameter values outlined in Appendix A. Although we observe evidence of stochastic noise in the average $\mathrm{P}-\mathrm{ABM}$ tip cell distributions at the two time points presented in Figs. 2(a) and 2(c), both densities are roughly constant in the $y$ direction for this choice of initial conditions and TAF field. Comparison of the two graphs reveals that tip cells in the $\mathrm{P}-\mathrm{ABM}$ travel to the right and spread out over larger spatial areas as time increases. Additionally, the maximum number of tip cells found on average in a particular lattice site decreases with time.

Figures 2(b) and 2(d) present the corresponding P-ABM stalk cell distributions. We observe that the average stalk cell solution for the P-ABM travels toward the right-hand boundary, up the TAF gradient. Furthermore, the average number of stalk cells found in lattice sites appears to decrease along the $x$ direction. Unlike the tip cell solution, however, the stalk cell density does not appear to be constant in the $y$ direction: near the top and bottom edges of the lattice, for instance, the stalk cell density appears to be greater than its value at other points in the domain interior. This is likely an edge effect due to the boundary conditions of the P-ABM: tip cells attempting to exit the domain are reflected back, but still produce stalk cells during this movement attempt. Hence, the total number of tip cells (and stalk cells) will be greater near the edges of the lattice than at other points in the domain interior. Further investigations reveal that a similar edge effect occurs at the left-hand boundary of the domain (results not shown).

Since such edge effects will likely lead to differences between the P-ABM distribution and the 2D snail-trail model solutions, we have decided to reduce their influence by initializing the $2 \mathrm{D}$ snail-trail model with $\mathrm{P}-\mathrm{ABM}$ data at time $t_{0}=0.2$, rather than $t_{0}=0$, to avoid simulating the snail-trail PDE too close to the boundary at $x=0$. Hence, solutions to the $2 \mathrm{D}$ snail-trail model were calculated for the time interval $t \in[0.2,2]$, rather than $t \in[0,2]$.

The values of $D, \chi, \lambda$, and $\beta_{n}$ in the continuous snail-trail model were determined from the $\mathrm{P}-\mathrm{ABM}$ parameter values (see Ref. [32] for details). Previous investigations of discrete 


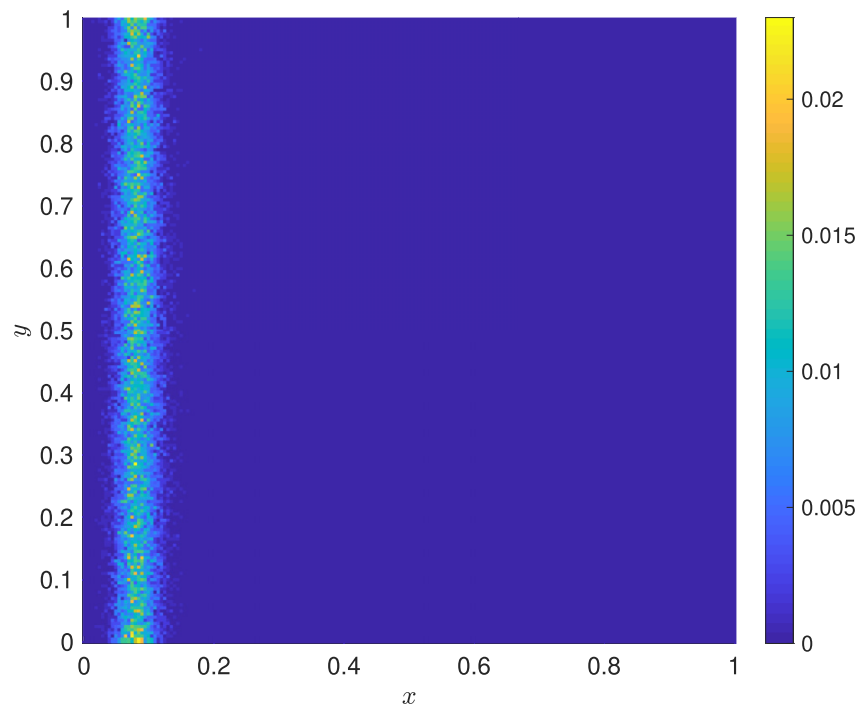

(a) Tip Cell Density, $t=0.2$

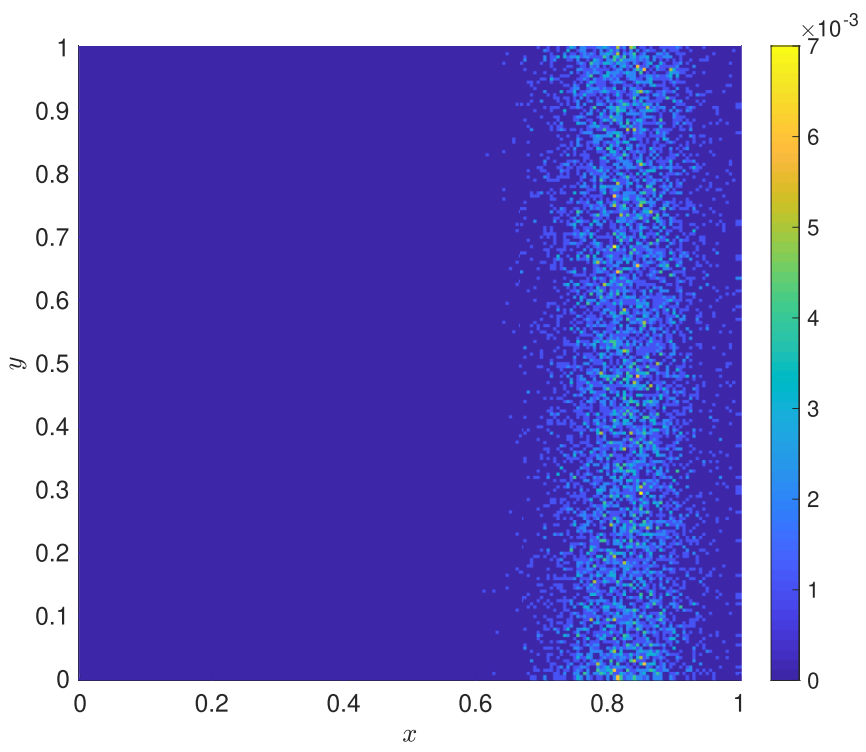

(c) Tip Cell Density, $t=2$

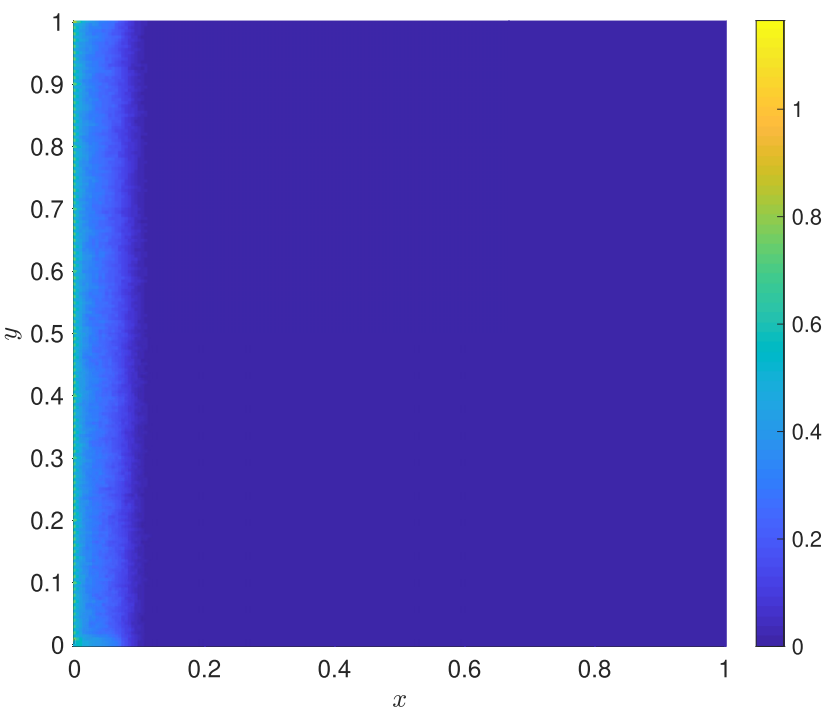

(b) Stalk Cell Density, $t=0.2$

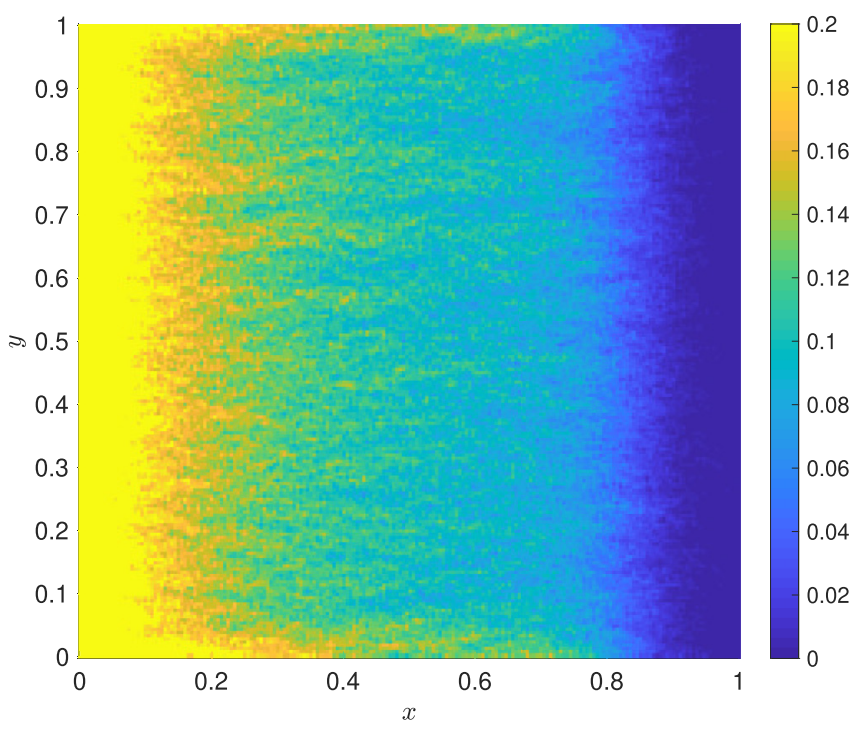

(d) Stalk Cell Density, $t=2$

FIG. 2. Heat maps of the average P-ABM (a),(c) tip cell and (b),(d) stalk cell solution over 1000 realizations at times (a),(b) $t=0.2$ and (c),(d) $t=2$. Color bars indicate the average number of cells that were located at that lattice point. The P-ABM parameter values and initial conditions are listed in Appendix A.

and continuum models $[32,66,67]$ have shown that the meanfield approximation used to construct the 2D snail-trail model can break down when anastomosis occurs. We correct for this potential breakdown by following the procedure outlined by Pillay et al. [32], where the value of $\beta_{e}$ in Figs. 3-5 is chosen to minimize the squared difference between 2D snail-trail and $\mathrm{P}-\mathrm{ABM}$ solutions at times $t=0.2,0.4, \ldots, 2$ (see Appendix B for details on the numerical methods). Since we have assumed that we do not need a corrective factor in this section, we set $\kappa(x, y)=1$.

Figure 3 presents tip and stalk cell solutions from the $2 \mathrm{D}$ snail-trail model at time $t=2$, using the parameter regime listed in Table I. We observe in Fig. 3(a) that the PDE tip cell solution appears to be approximately uniform in the $y$ direction and resembles that of the $\mathrm{P}-\mathrm{ABM}$. However, the dif- ferences between the two solutions are larger than one would expect from visual inspection: the $L_{2}$ norm of the difference between $\mathrm{P}-\mathrm{ABM}$ and $2 \mathrm{D}$ snail-trail results across all lattice sites is approximately equal to 0.10 when $t=2$, which is about $69 \%$ of the $L_{2}$ norm of $\mathrm{P}-\mathrm{ABM}$ results at that time point.

TABLE I. Continuum parameter values used to simulate the 2D snail-trail model given by Eqs. (3)-(5) in Figs. 3-5. Parameter values were determined from $\mathrm{P}-\mathrm{ABM}$ parameters based on expressions given by Pillay et al. [32].

\begin{tabular}{cccccc}
\hline \hline$D$ & $\chi$ & $\lambda$ & $\beta_{n}$ & $h$ & $\beta_{e}$ \\
\hline $10^{-3}$ & 0.4 & 0.16 & 160 & $200^{-1}$ & (see figure caption) \\
\hline \hline
\end{tabular}




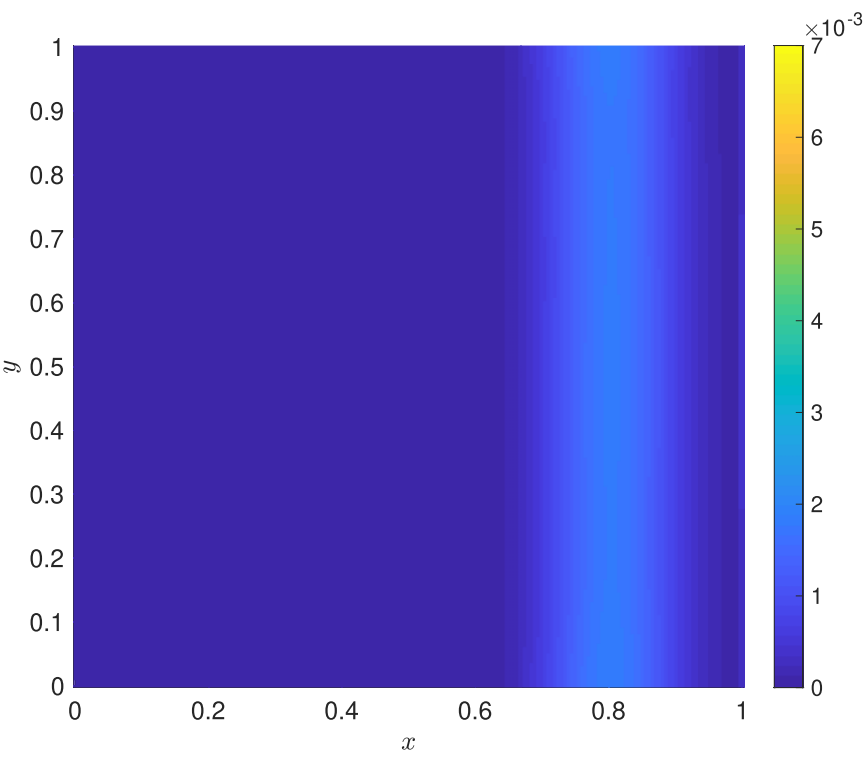

(a) Snail-Trail PDE, Tip Cell Density

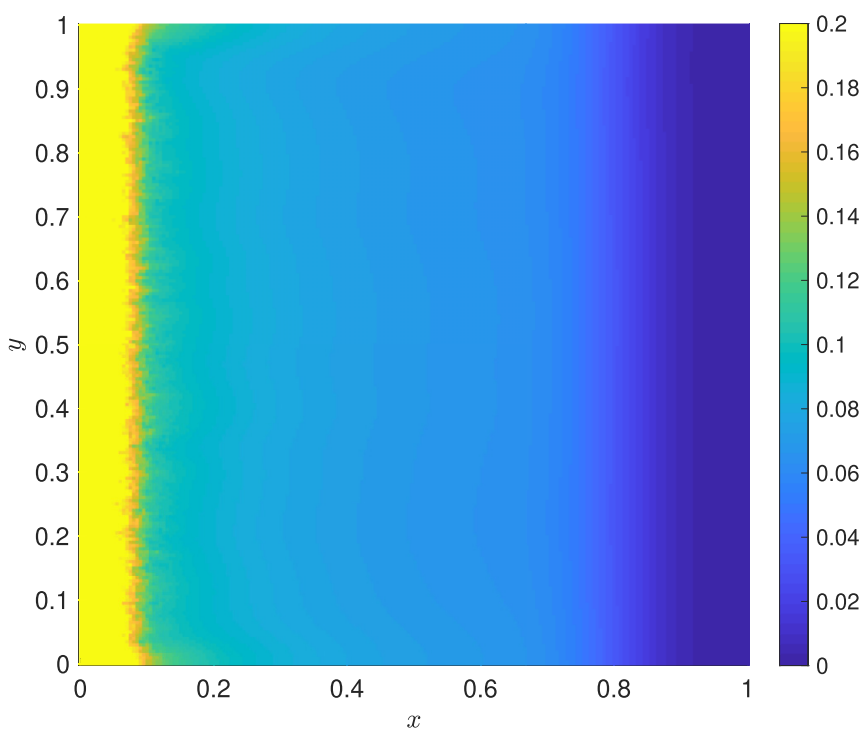

(b) Snail-Trail PDE, Stalk Cell Density

FIG. 3. Heat maps of the 2D snail-trail (a) tip and (b) stalk cell solution, subject to the TAF field $c(x, y)=x$, at $t=2$. Color bars indicate the cell density at $t=2$. Continuum parameters are listed in Table I, with $\kappa=1$. The parameter $\beta_{e}$ was fitted to the $\mathrm{P}-\mathrm{ABM}$ results using the numerical methods described in Appendix B $\left(\beta_{e}=4.65 \times 10^{-12}, 95 \% \mathrm{CI}\right.$ : [0, 0.123]). The snail-trail model was initialized at $t=0.2$ using the average $\mathrm{P}-\mathrm{ABM}$ distribution at that time point (see Fig. 2).

Figure 3(b) presents the corresponding 2D snail-trail stalk cell solution at time $t=2$. Although we observe that it decreases along the $x$ direction, just as the P-ABM stalk cell solution, comparison of this graph with Fig. 2(d) reveals discrepancies between the two solutions. For instance, in Fig. 3(b) the PDE stalk cell density appears to be constant in the $y$ direction, whereas the $\mathrm{P}-\mathrm{ABM}$ average stalk cell density is greater near the top and bottom boundaries. At $t=2$, the $L_{2}$ norm of the difference between the P-ABM and snail-trail stalk cell solutions across all lattice sites is 8.2, which is about $26 \%$ of the $L_{2}$ norm of the P-ABM results at that time point. Therefore the snail-trail model does not accurately capture the stalk cell density when there is no corrective factor.

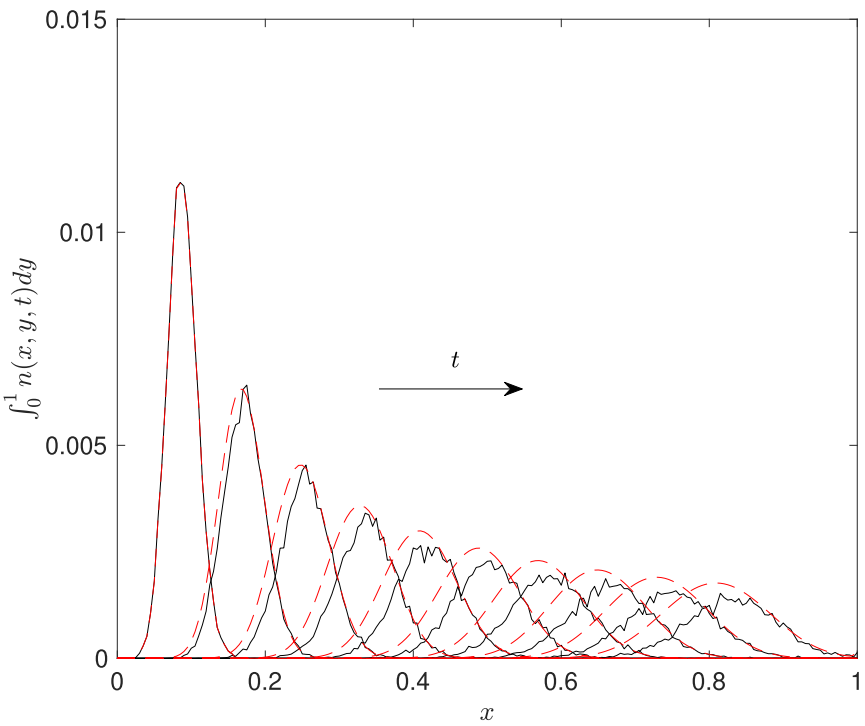

(a) Tip Cells

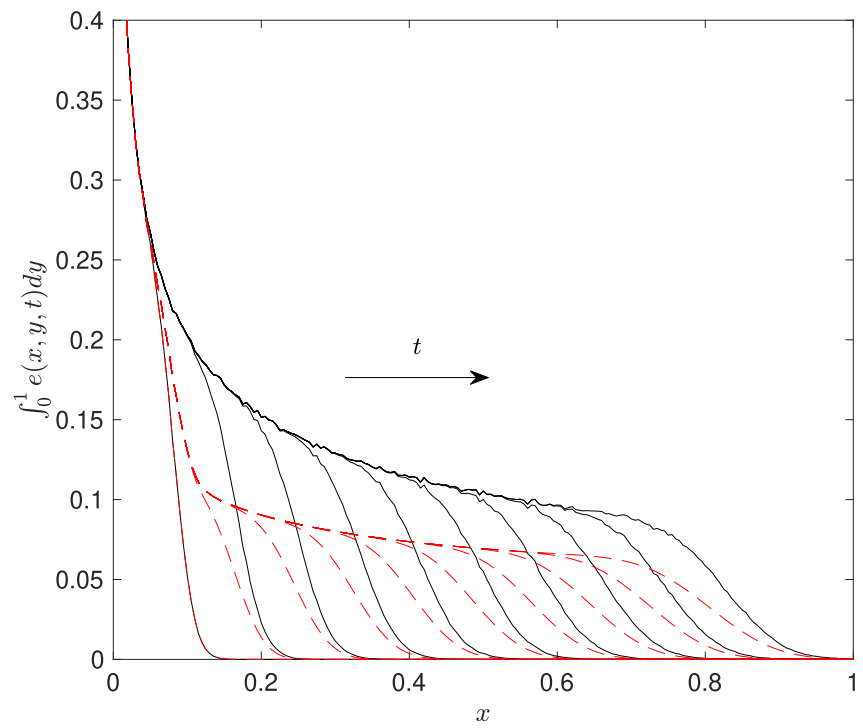

(b) Stalk Cells

FIG. 4. Solutions of the (a) tip cell and (b) stalk cell densities given by the P-ABM and 2D snail-trail model, column averaged in the $y$ direction, at $t=0.2,0.4, \ldots, 2$, with $\kappa(x, y)=1$. Key: P-ABM distribution (solid black lines); column-averaged 2D snail-trail solution (red dashed lines). For colors, we refer to the online article. Initial conditions and parameter values: as in Figs. 2 and 9. 


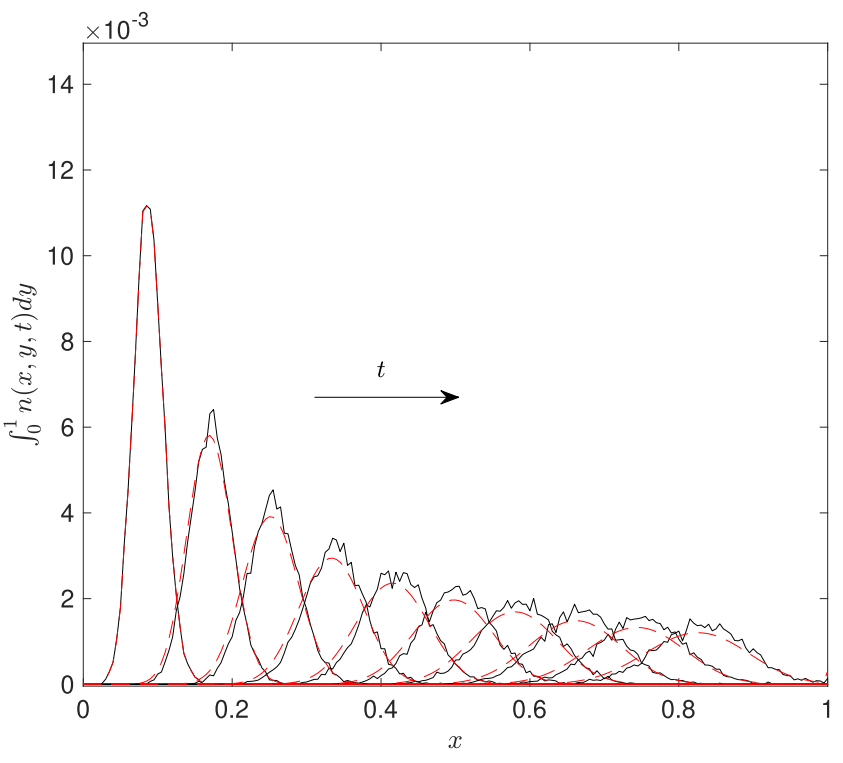

(a) Tip Cell Densities

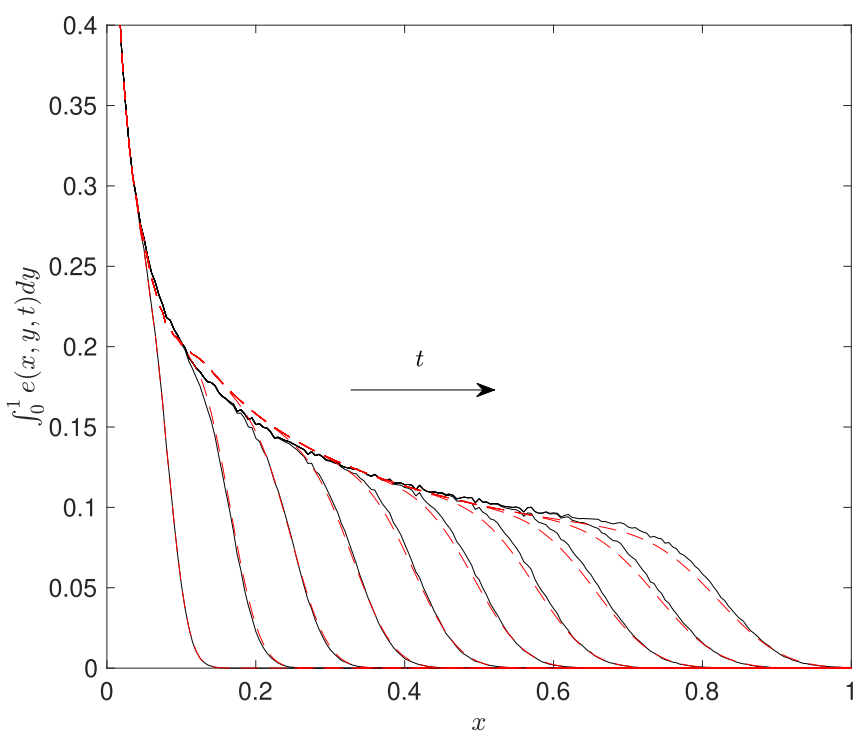

(b) Stalk Cell Densities

FIG. 5. Solutions of the (a) tip and (b) stalk cell densities given by the P-ABM and 2D snail-trail model, column averaged in the $y$ direction, at $t=0.2,0.4, \ldots, 2$, with $D=10^{-3}, \chi=0.4, \beta_{n}=160, \lambda=0.16$, and $\kappa(x, y)=2$. The parameter $\beta_{e}$ was fitted to the $\mathrm{P}-\mathrm{ABM}$ results using the numerical methods described in Appendix B ( $\beta_{e}=4.77,95 \%$ CI: [4.73, 4.82]). Key: P-ABM distribution (solid black lines); 2D snail-trail solution (red dashed lines). For colors, we refer to the online article. The PDE was initialized using P-ABM data at $t=0.2$ and simulated on the interval $t \in[0.2,2]$.

To better visualize the extent of differences between the discrete and continuum solutions, we numerically integrated the $2 \mathrm{D}$ results of each model in the $y$ direction (see Appendix B for details). The resulting column-averaged data are presented in Fig. 4. We observe in Fig. 4(a) that the columnaveraged snail-trail tip cell solutions appear to have the same bell curve profile as the $\mathrm{P}-\mathrm{ABM}$ solutions, and that their leading edges travel with roughly identical speeds (the speeds are within $3 \%$ of each other). However, the snail-trail model tip cell solution tends to overestimate that of the P-ABM. We conclude that while the snail-trail model captures many aspects of the $\mathrm{P}-\mathrm{ABM}$ tip cell distribution when $\kappa=1$, there are observable discrepancies between the two models.

Figure 4(b) presents the column-averaged $\mathrm{P}-\mathrm{ABM}$ and $2 \mathrm{D}$ snail-trail stalk cell densities. We observe that the snail-trail solution underestimates that of the P-ABM for all of the time points shown here. This underestimation persists even when we exclude possible edge effects by column averaging the 2D results over the smaller interval $y \in[0.05,0.95]$ (Supplemental Material Fig. 1 [68]). This suggests that the discrepancies between the snail-trail and $\mathrm{P}-\mathrm{ABM}$ are largely generated within the domain interior, rather than near the boundaries.

We have also found that even when branching and anastomosis events are neglected in the $\mathrm{P}-\mathrm{ABM}$ and $2 \mathrm{D}$ snail-trail model (so that $\lambda=\beta_{e}=\beta_{n}=0$ ), the column-averaged snailtrail stalk cell solution is always less than that of the P-ABM (Supplemental Material Fig. 2 [68]). In fact, the snail-trail stalk cell density is almost one-half the value of the $\mathrm{P}-\mathrm{ABM}$ results for this parameter regime.

These results demonstrate that when there is no corrective factor, the 2D snail-trail model underestimates the total stalk cell density and is not an accurate description of results from the $\mathrm{P}-\mathrm{ABM}$. We conclude that the stalk cell evolution should include a multiplicative factor $\kappa$, not necessarily equal to 1 , to approximate the $\mathrm{P}-\mathrm{ABM}$ stalk cell distribution well. Furthermore, the snail-trail model's underestimation of the P-ABM stalk cell density occurs even when branching or anastomosis are neglected, which is consistent with arguments from Secs. II and III.

TABLE II. TAF fields, gradient magnitudes, and values of $\kappa$ for the figures presented in Secs. V and VI. In all cases, $D=10^{-3}, \chi=0.4$, and $h=200^{-1}$. Except for Case 1, the other parameter values used in the snail-trail model were $\lambda=\beta_{e}=\beta_{n}=0$. Results for Case 3 are presented in Supplemental Material Figs. 4 and 5 [68].

\begin{tabular}{lccc}
\hline \hline Case & $c(x, y)$ & $\|\nabla c\|_{2}$ & $\kappa(x, y)$ \\
\hline 1 & $x$ & 1 & 2 \\
2 & $1-\left(x-\frac{1}{2}\right)^{2}-\left(y-\frac{1}{2}\right)^{2}$ & $2\left[\left(x-\frac{1}{2}\right)^{2}+\left(y-\frac{1}{2}\right)^{2}\right]^{1 / 2}$ & {$\left[\left(x-\frac{1}{2}\right)^{2}+\left(y-\frac{1}{2}\right)^{2}\right]^{-1 / 2}$} \\
3 & $x y$ & $\left(y^{2}+x^{2}\right)^{1 / 2}$ & $2\left(y^{2}+x^{2}\right)^{-1 / 2}$ \\
\hline \hline
\end{tabular}




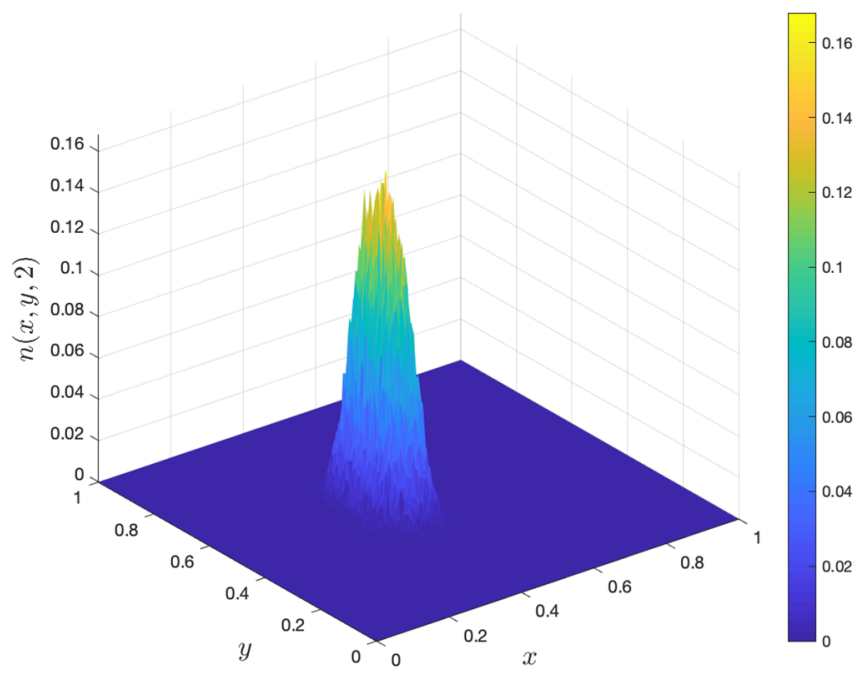

(a) 2D P-ABM Distribution, Tip Cells

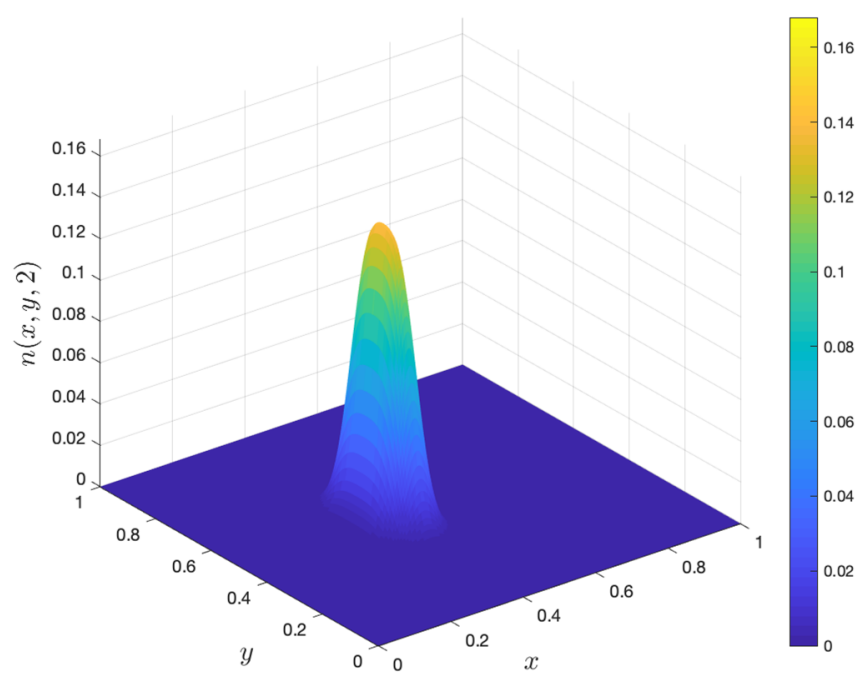

(c) 2D Snail-Trail Solution, Tip Cells

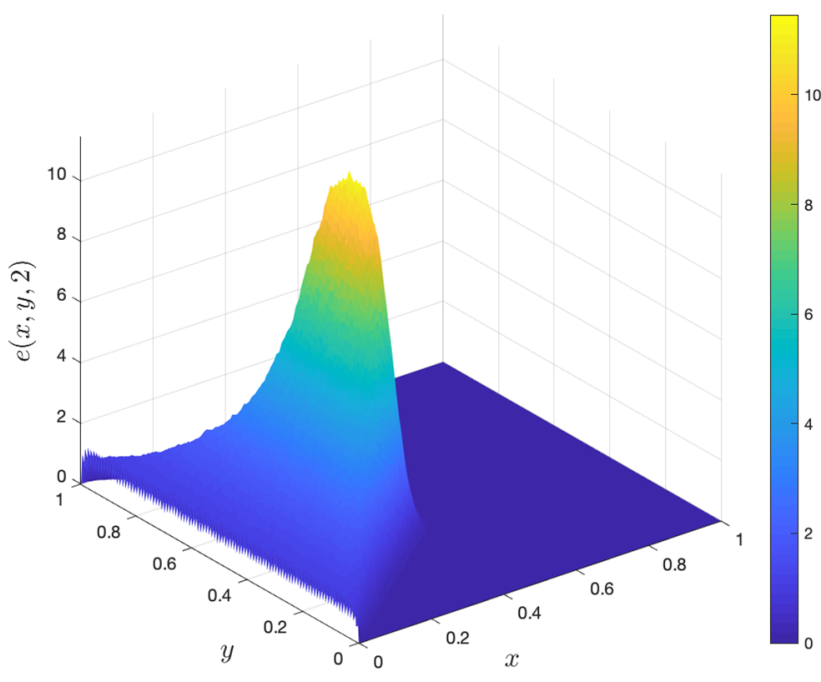

(b) 2D P-ABM Distribution, Stalk Cells

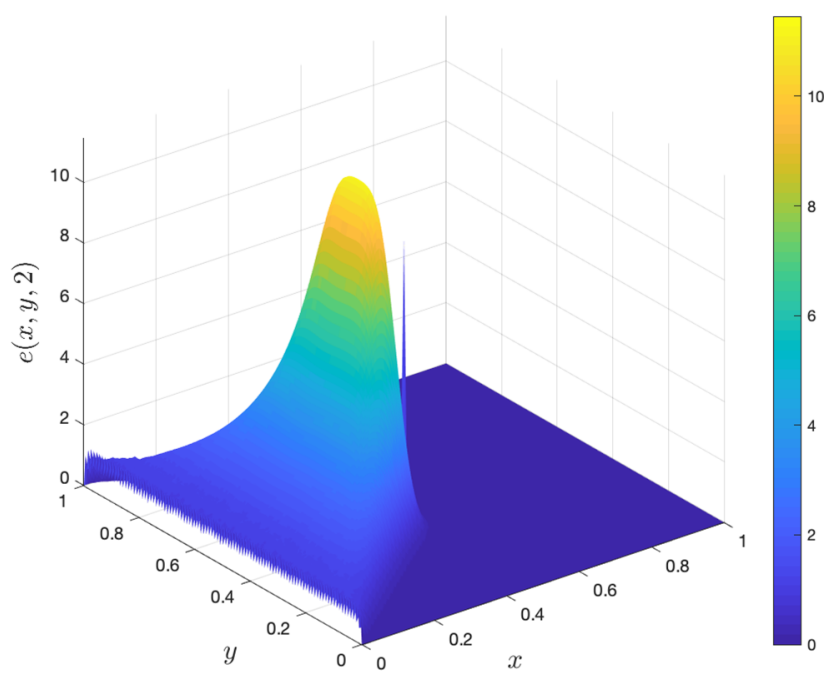

(d) 2D Snail-Trail Solution, Stalk Cells

FIG. 6. Results for the (a) P-ABM tip cell, (b) P-ABM stalk cell, (c) snail-trail tip cell, and (d) snail-trail stalk cell densities at $t=2$, subject to the TAF field $c(x, y)=1-(x-0.5)^{2}-(y-0.5)^{2}$. Parameter values: see Table II. The P-ABM used the same initial condition described in Appendix A, and its solution at $t=0.2$ was used to initialize the snail-trail PDE.

\section{VALIDATION OF THE SNAIL-TRAIL MULTIPLICATIVE FACTOR BY NUMERICAL SIMULATION}

Our results and earlier analysis suggest that including a multiplicative factor given by Eq. (14) in the 2D snail-trail stalk cell evolution equation will cause the continuum model to more accurately capture the $\mathrm{P}-\mathrm{ABM}$ solutions, provided tip cell movement is dominated by chemotaxis. In this section, we test this hypothesis by examining 2D snail-trail solutions for different TAF fields. The scenarios that we consider are presented in Table II.

We first examined the same case from Sec. IV, in which $c(x, y)=x$ (Case 1 in Table II). Using Eq. (14), we calculate $\kappa(x, y)=2$. Although the 2D snail-trail solution profiles (Supplemental Material Fig. 3 [68]) resemble those from
Fig. 3, further investigation reveals that, for both tip and stalk cell densities, the snail-trail model with $\kappa=2$ is a more accurate estimate of the average $\mathrm{P}-\mathrm{ABM}$ distribution.

To see this, we present the column-averaged tip and stalk cell solutions in Fig. 5. We observe in Fig. 5(a) that the snailtrail tip cell solution with $\kappa=2$ is a better approximation to the $\mathrm{P}-\mathrm{ABM}$ results than when $\kappa=1$ (the maximum pointwise difference between the two solutions is never more than $30 \%$ of the maximum tip cell density for the time points shown; furthermore, the total tip cell mass of the continuum and discrete models are within $4 \%$ of each other). Comparison of this graph with Fig. 4(a) further reveals that the snailtrail model with $\kappa=2$ overestimates the $\mathrm{P}-\mathrm{ABM}$ solution to a lesser extent than when $\kappa=1$. Figure $5(\mathrm{~b})$ presents the column-averaged stalk cell distributions and shows that 


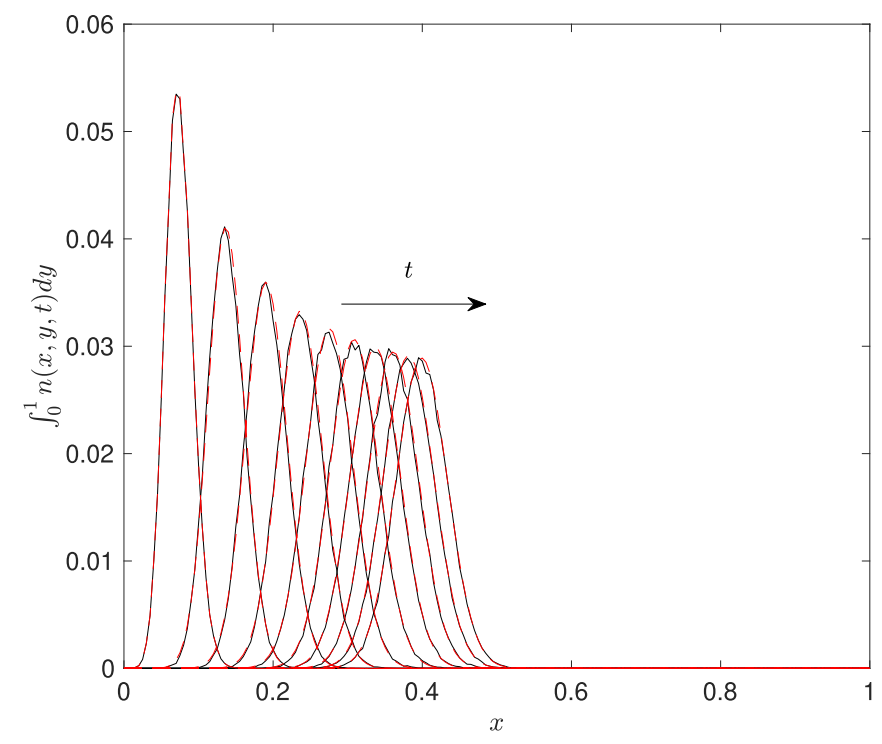

(a) Tip Cell Density

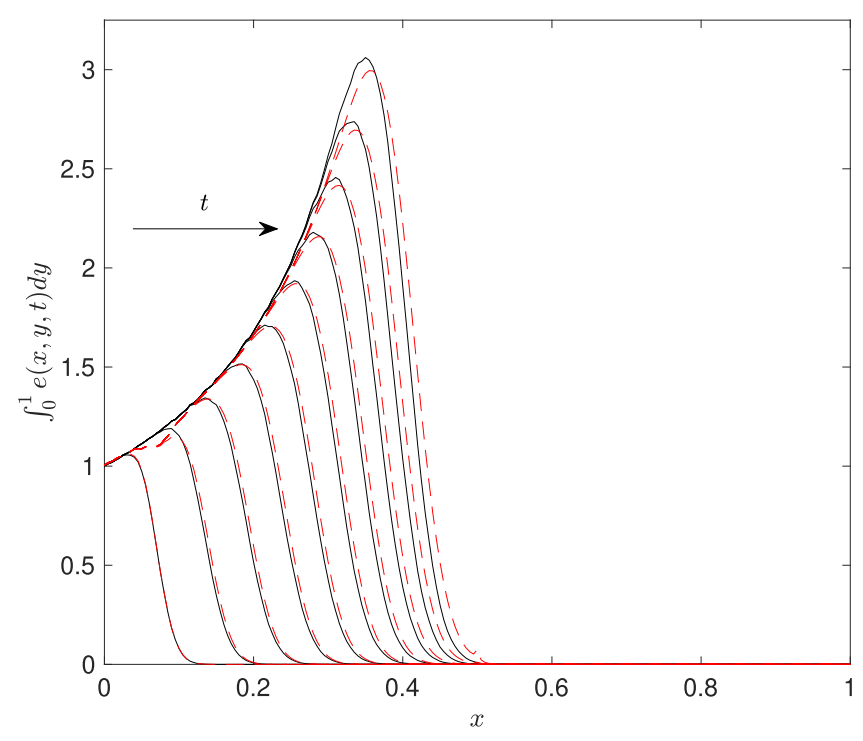

(b) Stalk Cell Density

FIG. 7. Results for the (a) tip, and (b) stalk cell densities from the P-ABM and 2D snail-trail model subject to the TAF field $c(x, y)=$ $1-(x-0.5)^{2}-(y-0.5)^{2}$, column averaged in the $y$ direction, at times $t=0.2,0.4, \ldots, 2$. Initial conditions and parameter values: as in Fig. 6. Key: P-ABM distribution (solid black lines), snail-trail solution (dashed red lines). For colors, we refer to the online article.

the discrepancy between the column-averaged $\mathrm{P}-\mathrm{ABM}$ and snail-trail solutions is also smaller than it was when $\kappa=1$. These observations indicate that our formula for $\kappa$ improves the agreement between tip and stalk cell solutions to the 2D snail-trail model and P-ABM.

In the aforementioned example, the chemotactic gradient varies in only one spatial dimension. However, our results can be extended to $2 \mathrm{D}$ chemotactic gradients. We have verified this for the TAF fields $c(x, y)=x y$ and $c(x, y)=1-(x-$ $0.5)^{2}-(y-0.5)^{2}$. For such simulations, we neglect branching and anastomosis because we have determined that neither process will affect the value of the multiplicative factor (in other words, for the remaining results presented in this section, $\lambda=\beta_{e}=\beta_{n}=0$ ).

Figures 6 and 7 presents results for the TAF field $c(x, y)=$ $1-(x-0.5)^{2}-(y-0.5)^{2}($ Case 2 in Table II), which attains a maximum value at the domain center. The corresponding 2D $\mathrm{P}-\mathrm{ABM}$ and snail-trail results at $t=2$ are presented in Fig. 6 . We observe in Fig. 6(a) that tip cells in the P-ABM have traveled from the left-hand boundary and have clustered near the point $(x, y)=(0.5,0.5)$, which is the location of the maximum TAF concentration. This tip cell behavior is expected, since the TAF gradient points toward the center of the domain. We also see in Fig. 6(b) that the density of stalk cells is much greater near the center of the domain than elsewhere. This is because the magnitude of the TAF gradient becomes small near $(x, y)=(0.5,0.5)$ : tip cells in the $\mathrm{P}-\mathrm{ABM}$ thus become more likely to move via random motion in this area, which in turn produces many stalk cells.

Figures 6(c) and 6(d) present the corresponding 2D snailtrail solutions for this TAF field. We observe that the snail-trail tip cell solution in Fig. 6(c) resembles that of the P-ABM, although there are some differences between the two sets of solutions due to stochastic effects (the $L_{2}$ norm of the difference between the tip cell solutions is within $11 \%$ of the
$L_{2}$ norm of the P-ABM distribution). Figure 6(d) reveals that the snail-trail model stalk cell solution profile also generally resembles that of the $\mathrm{P}-\mathrm{ABM}$ (the $L_{2}$ norms of the difference between the two solutions is also within $11 \%$ of the $L_{2}$ norm of the $\mathrm{P}-\mathrm{ABM}$ distribution). The most evident difference between the continuum and discrete stalk cell results occurs near the point $(x, y)=(0.5,0.5)$, where a "spike" of stalk cells arises because the value of $\kappa(x, y)$ blows up there.

This overestimation of the P-ABM stalk cell density can be perceived even when both sets of $2 \mathrm{D}$ solutions are column averaged in the $y$ direction (results are presented in Fig. 7). We find that the maximum difference between the columnaveraged tip cell solutions is less than $5 \%$ of the maximum tip cell density for the time points shown in Fig. 7(a). Furthermore, the total cell masses computed by the discrete and continuum models are within $1 \%$ of each other. Both of these results indicate that the snail-trail model accurately estimates the $\mathrm{P}-\mathrm{ABM}$ tip cell solution. However, there is greater discrepancy between the two models' column-averaged stalk cell solutions in Fig. 7(b). Although the total stalk cell mass given by the discrete and continuum models are very similar to each other (with approximately $1 \%$ difference), the maximum pointwise difference between the two solutions shown in the figure is on the order of $15 \%$ of the maximum stalk cell density. Such differences arise because our equation for $\kappa$ begins to blow up to infinity and overestimates the stalk cell density rate of change as the solution travels closer to $x=0.5$. This extra stalk cell production also explains why the column-averaged snail-trail stalk cell solution appears to travel a farther distance than that of the P-ABM in Fig. 7(b).

The numerical results thus corroborate our hypothesis that Eq. (14), which defines the multiplicative factor $\kappa$ as a function of space, causes the snail-trail model solutions to approximate $\mathrm{P}-\mathrm{ABM}$ results well (except in regions where the TAF gradient is close to zero). 
We conclude this section by testing whether it is possible to obtain similar agreement between the discrete and continuum models by taking $\kappa$ to be constant, in contrast to the more general formula given by Eq. (14). This test is inspired by previous investigators who employed this assumption in 1D snail-trail models and used ad hoc approaches to estimate $\kappa$ $[32,46]$. To determine if agreement between the two models can be attained when $\kappa$ is constant, we numerically solved the 2D snail-trail PDE with a value of $\kappa$ that minimized the squared difference between its results and those from the $\mathrm{P}-\mathrm{ABM}$ for the three TAF fields listed in Table II (numerical methods are listed in Appendix B). When the magnitude of the TAF gradient is constant, the optimal value of $\kappa$ is within $1 \%$ of the value predicted by Eq. (14) (results are presented in Supplemental Material Fig. 6 [68]). For more general TAF fields whose gradients vary in space, however (such as Cases 2 and 3 in Table II), we find that the discrepancies between snail-trail and $\mathrm{P}-\mathrm{ABM}$ results are greater when $\kappa$ is taken to be a constant value than in cases where its value is determined by Eq. (14) (results for the TAF field $c(x, y)=1-(x-0.5)^{2}-(y-0.5)^{2}$ are presented in Supplemental Material Fig. 7 [68]). We conclude that, for general TAF fields whose gradients vary in space, the multiplicative factor that causes the snail-trail model to best approximate $\mathrm{P}-\mathrm{ABM}$ solutions is not necessarily constant, in contrast to previous ad hoc approaches for estimating this factor.

\section{DERIVATION OF THE 1D SNAIL-TRAIL MODEL}

While we have shown that the 2D snail-trail model presented in Sec. II can be in good agreement with the P-ABM, the relationship between our continuum model and other 1D snail-trail models in the literature remains unclear. Such 1D systems ostensibly describe the dynamics of tip and stalk cell densities that have been column averaged in the direction perpendicular to that of the moving tip cells [46,47], but it is unknown what assumptions are required to ensure that this interpretation is appropriate. To address these open questions, we formally reduce our $2 \mathrm{D}$ snail-trail system to one spatial variable by deriving the dynamics that govern its columnaveraged solutions and compare them to existing 1D snail-trail models.

To do this, we define the new column-averaged dependent variables

$$
\begin{aligned}
N(x, t) & =\int_{0}^{1} n(x, y, t) d y, \\
E(x, t) & =\int_{0}^{1} e(x, y, t) d y, \\
C(x) & =\int_{0}^{1} c(x, y) d y .
\end{aligned}
$$

Integrating Eqs. (3) and (4) with respect to $y$, and applying the boundary conditions given by Eq. (5), leads to the equation

$$
\begin{gathered}
N_{t}=\int_{0}^{1} D n_{x x}-\chi\left(n c_{x}\right)_{x} d y+\lambda \int_{0}^{1} n c d y \\
-\beta_{e} \int_{0}^{1} n e d y-\beta_{n} \int_{0}^{1} n^{2} d y
\end{gathered}
$$

$$
E_{t}=\frac{1}{h} \int_{0}^{1} \kappa(x, y)\|D \nabla n-\chi n \nabla c\|_{2} d y .
$$

We remark that subscript notation has been used to indicate partial derivatives in Eqs. (15) and (16). For general TAF fields and tip/stalk cell solutions, the above system cannot be reduced further. However, under the following assumptions it is possibly to simplify both equations in terms of one spatial variable. We first make a mean-field approximation in the tip cell evolution equation, in which it is assumed

$$
\begin{aligned}
& \int_{0}^{1} n c d y \approx\left[\int_{0}^{1} n d y\right] \times\left[\int_{0}^{1} c d y\right]=N C, \\
& \int_{0}^{1} n e d y \approx\left[\int_{0}^{1} n d y\right] \times\left[\int_{0}^{1} e d y\right]=N E \\
& \int_{0}^{1} n^{2} d y \approx\left[\int_{0}^{1} n d y\right] \times\left[\int_{0}^{1} n d y\right]=N^{2} .
\end{aligned}
$$

Next, we assume that the quantities $n, n_{x}, n_{x x}, c_{x}$, and $c_{x x}$ are independent of $y$. Combining this assumption with the meanfield approximation reduces the first integral in Eq. (15) to $D N_{x x}-\chi\left(N C_{x}\right)_{x}$.

The stalk cell evolution equation can also be simplified to one spatial variable under the following assumptions: We consider the $y$ component of the tip cell net flux $\left(D n_{y}-\chi n c_{y}\right)$ to be negligible, and we assume that a mean-field approximation is valid, such that the stalk cell evolution equation reduces to

$$
E_{t} \approx \frac{1}{h} \int_{0}^{1} \kappa(x, y) d y \times \int_{0}^{1}\left|D n_{x}-\chi n c_{x}\right| d y .
$$

If the TAF field varies in the $y$ direction, then the first integral may diverge: for example it is undefined for the TAF field $c(x, y)=1-(x-0.5)^{2}-(y-0.5)^{2}$. We therefore redefine the column-averaged multiplicative factor, $\widetilde{\kappa}(x)$, in terms of the column-averaged TAF field $C(x)$ using a similar formula to Eq. (14):

$$
\widetilde{\kappa}(x) \approx \frac{4 D}{h \chi\left|C_{x}\right|} .
$$

For the 2D TAF fields $c(x, y)=x, c(x, y)=0.5(x+y)$ and $c(x, y)=1-(x-0.5)^{2}-(y-0.5)^{2}$, we have found that using Eq. (19) causes the 1D snail-trail model to become a good approximation of column-averaged 2D snail-trail solutions in regions where the TAF gradient is nonzero (see Supplemental Material Figs. 8-10 [68]).

If we also assume that the sign of $\left(D n_{x}-\chi n c_{x}\right)$ does not change in the domain, then we may bring the second integral in Eq. (18) into the absolute value function. Since we have already assumed that a mean-field approximation is valid and that $n, n_{x}$, and $c_{x}$ do not vary in the $y$ direction, this means that the second integral simplifies to $\left|D N_{x}-\chi N C_{x}\right|$.

So long as the above assumptions hold, the $1 \mathrm{D}$ version of Eqs. (3)-(5) is

$$
\frac{\partial N}{\partial t}=D \frac{\partial^{2} N}{\partial x^{2}}-\chi \frac{\partial}{\partial x}\left(N \frac{\partial C}{\partial x}\right)+\lambda N C-\beta_{e} N E-\beta_{n} N^{2},
$$




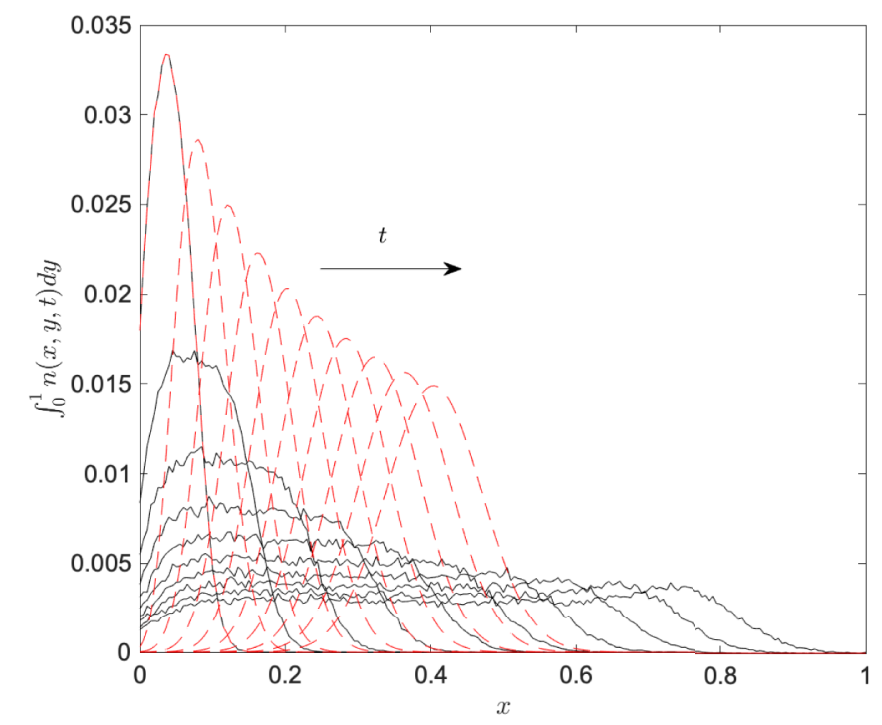

(a) Tip Cell Density

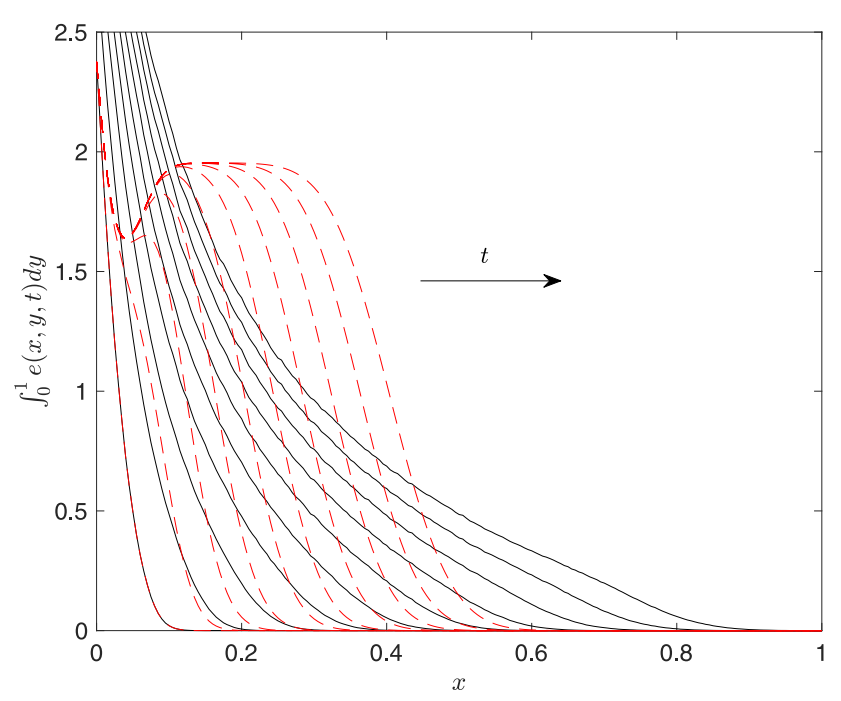

(b) Stalk Cell Density

FIG. 8. Solutions for the (a) tip and (b) stalk cell densities given by the $1 \mathrm{D}$ snail-trail model and the P-ABM at $t=0.2,0.4, \ldots, 2$. The $\mathrm{P}-\mathrm{ABM}$ is subject to the TAF field $c(x, y)=x y$, and its results have been column averaged in the $y$ direction; the 1D snail-trail model is subject to the column-averaged TAF field $[C(x)=0.5 x]$, such that $\widetilde{\kappa}(x)=4$. Key: column-averaged $\mathrm{P}-\mathrm{ABM}$ distribution (solid black lines), 1D snail-trail solution (dashed red lines). Parameter values: as in Table II. The P-ABM uses the same initial condition outlined in Appendix A, while the $1 \mathrm{D}$ snail-trail model was initialized with the column-averaged $\mathrm{P}-\mathrm{ABM}$ results at $t=0.2$. For colors, we refer to the online article.

$$
\begin{gathered}
\frac{\partial E}{\partial t}=\frac{\widetilde{\kappa}(x)}{h}\left|D \frac{\partial N}{\partial x}-\chi N \frac{\partial C}{\partial x}\right| \\
D \frac{\partial N}{\partial x}-\chi N \frac{\partial C}{\partial x}=0 \text { at } x=0,1
\end{gathered}
$$

where $\widetilde{\kappa}(x)$ is defined by Eq. (19).

When $c(x, y)=x$, we find that $\widetilde{\kappa}(x)=2$ and the 1D snailtrail model is equivalent to the one used by Pillay et al. [32]. Additionally, the snail-trail model from Byrne and Chaplain [47] may be recovered if one sets $\beta_{n}=0$ and $\widetilde{\kappa}(x)=1$, includes an additional term in Eq. (20) to account for tip cell production due to branching from stalk cells, and introduces a term in Eq. (21) to account for stalk cell elimination due to vessel regression. We conclude that Eqs. (20)-(22) are consistent with existing 1D snail-trail models under the assumptions listed above. It follows that the assumptions made in deriving Eqs. (20)-(22) also apply to existing 1D snail-trail models of angiogenesis.

It is evident from our derivation that numerous conditions must be satisfied to ensure that the 1D system given by Eqs. (20)-(22) is in good agreement with column-averaged 2D snail-trail solutions. In spite of this, we have found that there are certain TAF fields, such as $c(x, y)=C(x)=x$, for which this does occur, and for which the 1D snail-trail solutions are accurate approximations to column-averaged $\mathrm{P}-$ ABM data (Supplemental Material Figs. 8 and 9 [68]).

For 2D TAF fields whose gradients vary significantly with respect to space, however, many of the above assumptions are likely to break down and the 1D snail-trail model will not capture column-averaged $\mathrm{P}-\mathrm{ABM}$ data well. In the case where $c(x, y)=x y$, for instance, we violate the assumptions that $c_{x}$ is independent of $y$ and that the $y$ component of net tip cell flux is negligible compared to its $x$ component.
Figure 8 presents column-averaged $\mathrm{P}-\mathrm{ABM}$ results subject to the TAF field $c(x, y)=x y$, along with 1D snail-trail model solutions generated using the column-averaged TAF field $C(x)=0.5 x$ (Case 3 in Table II). We observe that the snail-trail tip and stalk cell densities are poor approximations to the $\mathrm{P}-\mathrm{ABM}$ results for the time points shown: for example, the $\mathrm{P}-\mathrm{ABM}$ tip and stalk cells appear to travel with a faster speed than the 1D snail-trail solutions. This is due to the difference between the 2D TAF field and its column-averaged version. Since tip cells in the snail-trail model and P-ABM move faster in larger TAF gradients, and the $x$ component of the $2 \mathrm{D}$ TAF gradient is equal to $y$, tip cells in the 2D P-ABM are more likely to travel right when they are near the top boundary of the domain. We confirmed this by visualizing the P-ABM tip cell solutions in 2D (Supplemental Material Fig. 4 [68]). The column-averaged TAF field $[C(x)=0.5 x]$, however, ignores any spatial variation of the TAF with respect to $y$, hence the $1 \mathrm{D}$ snail-trail model ignores these possible differences in tip cell speeds. We conclude that when the difference between the 2D TAF field and its column-averaged version is sufficiently large, the $1 \mathrm{D}$ snail-trail model will be a poor approximation to column-averaged $\mathrm{P}-\mathrm{ABM}$ results.

\section{DISCUSSION AND CONCLUSION}

In this article, we have studied a simple, phenomenological continuum framework for 2D network formation that contains a core snail-trail assumption, in which follower stalk cells proliferate along the path of leader tip cells. By investigating cell dynamics within a domain that contains a generic chemoattractant at steady state, we reasoned that a multiplicative factor must be introduced in the stalk cell rate equation to account for the subtleties of multidirectional cell movement. Our derivation of this factor, which is based on 
physical principles, demonstrates that snail-trail models are valid descriptions of tip and stalk cell dynamics when their movement is dominated by chemotaxis. Numerical solutions to the revised snail-trail model are in good agreement with ensemble average distributions from an existing discrete model, which also utilizes the snail-trail assumption [32]. We have thus identified a crucial, but heretofore implicit, condition that must be satisfied for the snail-trail modeling framework to accurately describe leader-follower behavior.

Similar implicit assumptions were identified during our derivation of the 1D column-averaged version of our snailtrail model, which is equivalent to several existing descriptions of network formation $[32,46,47]$. Namely, we found that this 1D model is an appropriate description of (columnaveraged) $\mathrm{P}-\mathrm{ABM}$ results when a mean-field approximation is valid and when the differences between the 2D TAF field and its column-averaged counterpart are sufficiently small. Our numerical results indicate that when such assumptions are violated, the 1D snail-trail model (unlike the 2D snail-trail model) cannot describe the discrete model's solutions well.

Our analysis suggests that certain metrics for cell migration, such as the average cell displacement, could indicate when cell movement is dominated by chemotaxis and, hence, when snail-trail models would likely be accurate descriptions of biological experiments for network formation. In future work, we aim to explore the success of these metrics in anticipating how well snail-trail models describe such data.

Before engaging in this research, however, several possible extensions should be made to the current snailtrail model so as to improve its applicability to experiments. For instance, we assumed in this article that all sources of cell motion (besides chemotaxis) could be modeled as an isotropic random motion term in the PDE system and P-ABM. Although this is a common modeling choice, there are certain cells whose motion cannot be described in this fashion [69]; other types of cell movement terms should therefore be considered and evaluated experimentally, within the particular biological context being studied (e.g., in angiogenesis), before using the equations used here. Furthermore, we analyzed tip and stalk cell dynamics in an idealized condition where the TAF concentration was at steady state. In more biologically relevant scenarios, however, the TAF field is unlikely to match the profiles investigated in Table II and its dynamics will likely be more complex: for example, cells may degrade the chemoattractant and create cell-induced gradients [57]. It may therefore be necessary to include such dynamics in the snail-trail modeling framework when it is used to analyze experimental results.

Another improvement to the snail-trail modeling framework is to determine how the corrective factor's expression changes in response to different boundary conditions. Since our focus in this article was to evaluate the accuracy of the snail-trail model within the domain interior, we did not resolve the "edge effects" in Secs. IV and V that contributed to differences between the P-ABM and 2D snail-trail solutions. Understanding how to correct for such boundary conditions, however, will be required if snail-trail models are used to investigate experimental data, and we will address this lack of insight in future work.
Although the corrective factor derived in this article is new to the 2D snail-trail system discussed in Sec. II, similar expressions have appeared in other mathematical descriptions of network morphogenesis that employ different modeling frameworks. For example, a term nearly identical to Eq. (14) appears in a hybrid ABM for angiogenesis, even though that particular model does not use the net tip cell flux to calculate capillary densities [70,71]. This similarity does not arise by chance: indeed, we show in Appendix $\mathrm{C}$ that one can rederive Eq. (14) by employing the procedure outlined by Vilanova et al. [71]. The equivalence between the two expressions results from a shared physical assumption underlying both modeling frameworks: that new sprouts proliferate along the path of moving tip cells. Since each corrective factor serves to account for the total sprout density created by this mechanism, it is therefore expected that they will be identical. Hence, our work has identified how a core physical assumption of trail-following behavior translates into a corrective factor is conserved across distinct modeling frameworks, and we expect this factor to appear in other mathematical descriptions of biological processes that also include a snail-trail assumption.

This raises an interesting question of whether it is possible to identify other core physical mechanisms from mathematical descriptions for network formation, for instance by investigating the relationship between phenomenological snail-trail models and other continuum systems for network formation that have been based on first principles. Such "coarse-grained" models have garnered increasing attention in recent years, as they are derived directly from discrete models and describe their average solution in time and/or space $[35,49,72]$. Coarse-grained systems can be used to (indirectly) analyze their underlying discrete models in a tractable manner: for example, they may determine if solutions are sensitive to certain parameters. However, coarse-grained systems tend to be highly nonlinear, with the level of complexity depending on the rules of the discrete model $[49,66,73,74]$. For example, one may derive a coarse-grained PDE system from the $\mathrm{P}-\mathrm{ABM}$ (which we call the P-PDE). The P-PDE contains more nonlinear terms than the snail-trail model and has a very different stalk cell evolution equation. Nevertheless, Pillay et al. identified in [32] certain parameter regimes for which solutions to the P-PDE were indistinguishable from those of a 1D snail-trail model that we showed is equivalent to the one derived in Sec. VI. In future work, we aim to use the results in this article to explain this observation and determine other regions of parameter space for which solutions to the two continuum models are indistinguishable from each other.

\section{ACKNOWLEDGMENTS}

W.D.M. acknowledges funding from the Keasbey Memorial Foundation and the University of Oxford Mathematical Institute (postgraduate scholarship). We also thank our anonymous reviewers for helpful feedback and suggestions that have improved the quality of the manuscript.

\section{APPENDIX A: REVIEW OF THE P-ABM}

In this section, we review the rules for the discrete model originally described in Pillay et al. [32] for the reader's 
TABLE III. TAF field and parameter values used in P-ABM simulations. All values are dimensionless.

\begin{tabular}{lccccccc}
\hline \hline$c(x, y)$ & $N$ & $h$ & $k$ & $P_{m}$ & $P_{p}$ & $\Delta t$ & $t_{\text {final }}$ \\
\hline (See figure caption) & 201 & $200^{-1}$ & 100 & 1 & $10^{-3}$ or 0 & $1 / 160$ \\
\hline \hline
\end{tabular}

reference. For a more detailed discussion of the $\mathrm{P}-\mathrm{ABM}$ and its implementation, we refer to the original article.

The domain of the $\mathrm{P}-\mathrm{ABM}$ is the unit square $(x, y) \in$ $[0,1]^{2}$. The lattice is equally spaced with $N$ points in both the $x$ and $y$ directions with a spatial step size $h$, such that $(N-1) h=1$. We denote lattice sites with indices $i, j$, where $0 \leqslant i, j \leqslant(N-1)$ and $\left(x_{i}, y_{j}\right)=(i h, j h)$. We assume that initially there is a blood vessel located at $x=0$ and that there is a TAF field at steady state, such that it may be represented as a continuous function $c(x, y)$.

We distinguish two cell types: tip cells and stalk cells. Tip cells can move and branch within the lattice, whereas stalk cells are created as a result of tip cell movement and tip-to-tip anastomosis. At each time step, tip cells, chosen uniformly at random with replacement, move with probability $P_{m}$. When a tip cell moves, its direction is chosen based on the probabilities listed in Eqs. (7) and (8) of Sec. III.

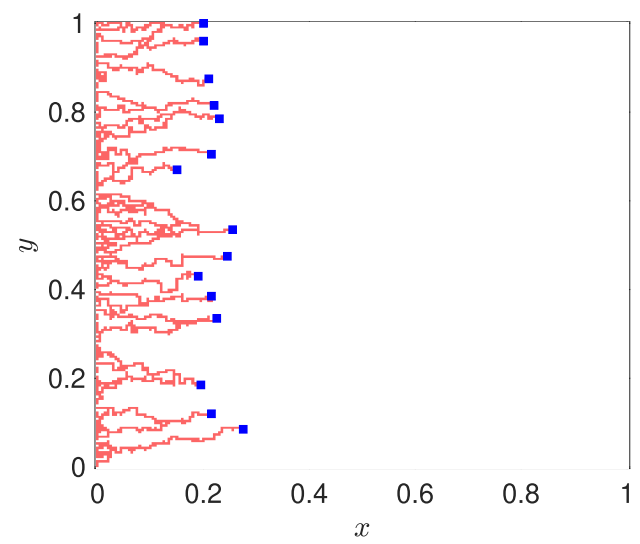

(a) $t=0.5$

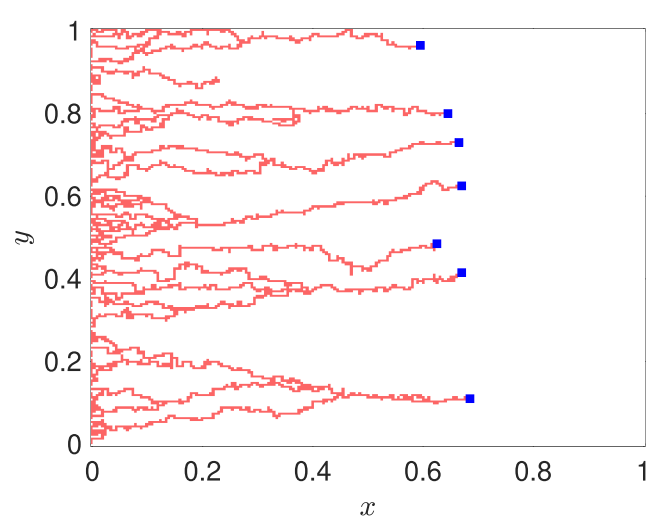

(c) $t=1.5$
Every time a tip cell moves from location $\left(x_{i}, y_{j}\right)$, a stalk cell is created at $\left(x_{i}, y_{j}\right)$. No-flux boundary conditions are applied along the edges of the lattice, such that if a tip cell attempts to move out of the lattice, then it is reflected back to its original position and a stalk cell is placed at the lattice site.

If a tip cell moves into a site occupied by at least one tip cell, then tip-to-tip anastomosis occurs with probability 1: All tip cells at this site are converted into a single stalk cell (i.e., $\mathrm{TC}+\mathrm{A} \cdot \mathrm{TC} \rightarrow \mathrm{EC}$, where $\mathrm{A} \geqslant 1$ ). We remark that the branching rules of our model allow for multiple tip cells to occupy a single lattice site (see below). If a tip cell instead encounters a stalk cell (tip-to-sprout anastomosis), then the tip cell is annihilated. Additionally, tip-to-tip anastomosis takes precedence over tip-to-sprout anastomosis. Following Pillay et al. [32], tip cells cannot anastomose with a stalk cell from the same sprout (i.e., self-loop formation is impossible), provided the tip cell has been at that site within

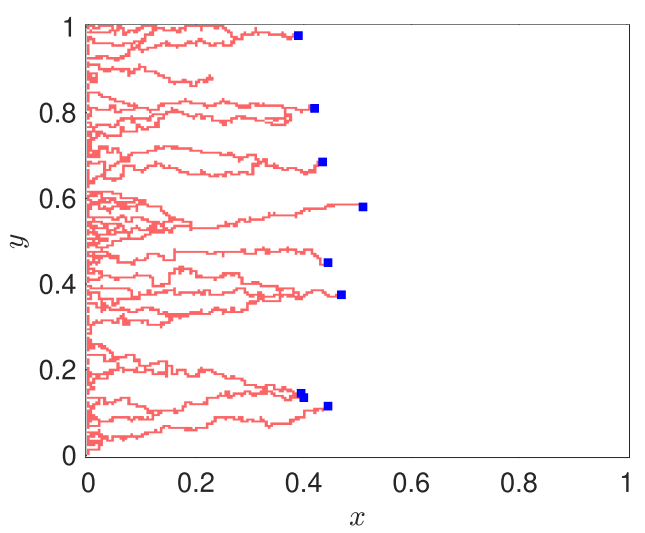

(b) $t=1$

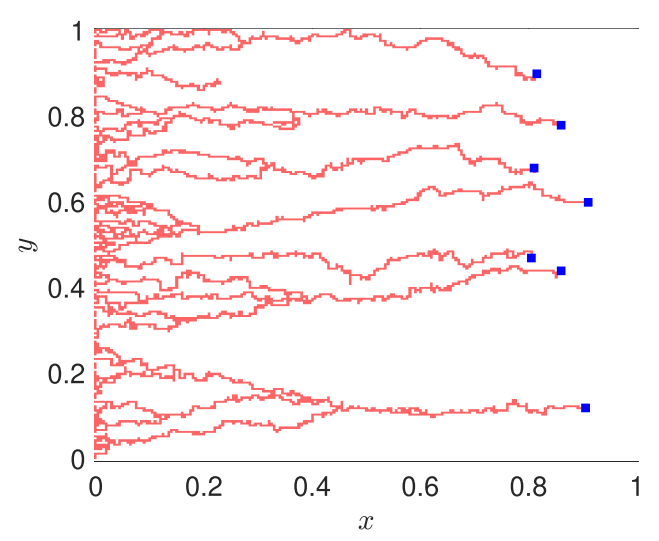

(d) $t=2$

FIG. 9. Network given by a single realization of the P-ABM, subject to the TAF field $c(x, y)=x$, at (a) $t=0.5$, (b) $t=1$, (c) $t=1.5$, and (d) $t=2$. Tip cells are dark blue squares, while stalk cells are in light red squares. Initial and boundary conditions: See Appendix A text. Parameter values: See Table III. For colors, we refer to the online article. 
10 prior movement steps. This constraint prevents tip cell extinction.

Branching is implemented in the following manner: tip cells proliferate with probability $P_{b}$, which is assumed to be proportional to the TAF concentration at $\left(x_{i}, y_{j}\right)$ :

$$
P_{b}=P_{p} c\left(x_{i}, y_{j}\right)
$$

where $P_{p}$ is a nonnegative constant chosen with the restriction that $P_{b} \leqslant 1$.

In the $\mathrm{P}-\mathrm{ABM}$, branching only occurs in the $y$ direction. Thus, if a tip cell at $\left(x_{i}, y_{j}\right)$ is chosen to branch, then this cell is removed and two daughter tip cells are created at $\left(x_{i}, y_{j \pm 1}\right)$. We note that no stalk cells are created when a tip cell branches. When a tip cell branches, its daughter cells are unable to branch during that time step. In practice, a tip cell may deposit a daughter cell in a site that is already occupied by another cell, which (combined with our self-loop restriction) means that more than one cell may simultaneously occupy a lattice site.

At time $t=0$, the domain is devoid of stalk cells while tip cells are placed at alternating sites along the left edge of the lattice, so tip cells are located initially at $(x, y)=(0,2 h)$, $(0,4 h), \ldots[0,(N-1) h]$. This initial condition is inspired by earlier investigations which showed that intercellular signaling creates a "salt-and-pepper" patterning of tip and stalk cells, in which tip cells are separated by no more than two stalk cells, before they migrate toward the TAF source [75].

One thousand realizations of the $\mathrm{P}-\mathrm{ABM}$ were simulated using the parameter values indicated in Table III. For a review of the algorithm used to find realizations of the P-ABM, we refer to Appendix B.

Figure 9 shows a network of tip and stalk cells created by a typical realization of the $\mathrm{P}-\mathrm{ABM}$ using the initial conditions, parameter values, and boundary conditions listed above at several time points. We observe multiple anastomosis events (especially near the left boundary of the lattice) and few branching events in this network. Figure 2 in Sec. IV shows the average tip and stalk cell distributions generated by the $\mathrm{P}-\mathrm{ABM}$ at $t=0.2$ and $t=2$, using the same parameter values and TAF field.

\section{APPENDIX B: NUMERICAL METHODS}

The algorithm used to generate a solution of the $\mathrm{P}-\mathrm{ABM}$ is given in Pillay et al. [32] and reproduced here for reference:

Choose $\Delta t$, the time step, and $t_{\text {final }}$, the time at which to terminate the P-ABM solution. Set $t=0$.

While $t<t_{\text {final }}$ and tip cells have not reached $i=N-1$ (the right edge of the lattice):

(1) Choose $N_{T C}$ tip cells at random with replacement, where $N_{T C}$ is the current number of tip cells.

Loop 1: For 1 to $N_{T C}$

(a) Choose a random number $r_{1} \in[0,1]$.

(b) If $r_{1} \leqslant P_{m}$, then the tip cell moves:

(i) Choose a random number $r_{2} \in[0,1]$.

(ii) The tip cell moves according to the probabilities $P_{x^{ \pm}}, P_{y^{ \pm}}$in Eqs. (7) and (8) of Sec. III, and a stalk cell is left behind. (iii) If tip-to-tip anastomosis can occur and the target site is occupied by one (or more) tip cells, then all tip cells at the lattice site are removed and a stalk cell is placed there.

(iv) Otherwise, if tip-to-sprout anastomosis can occur, then the target site is occupied by a stalk cell, and the stalk cell does not belong to the same sprout as the tip cell, then the tip cell is removed.

(v) Otherwise, the tip cell remains at the target site. End Loop 1

(2) Choose $N_{T C}$ tip cells at random with replacement, where $N_{T C}$ is the number of tip cells after completing Loop 1 .

Loop 2: For 1 to $N_{T C}$ :

(a) Choose a random number $r_{3} \in[0,1]$.

(b) If branching can occur and $r_{3} \leqslant P_{b}$, then the tip cell is removed and two cells are placed at adjacent lattice sites in the $y$ direction.

End Loop 2

(3) Adjust time $t=t+\Delta t$.

End While loop

The 2D PDE models given by Eqs. (3)-(5) were solved using the finite element method, implemented with the DOLFIN/FENICS library in Python [76-78]. The PDEs were solved on the unit square for $t \in[0.2,2]$ using $200 \times$ 200 second-degree continuous Lagrange finite elements. A Crank-Nicolson scheme with a time step of 0.001 was used to approximate the time derivative. The SCIPY library's NearestNDInterpolator function was used to set up a suitable initial condition on the mesh from the P-ABM solution at $t=0.2$. Column-averaged snail-trail solutions in the $y$ dimension were obtained using MATLAB's trapz function, which implements a numerical trapezoidal integration scheme.

Nonlinear least squares parameter fitting for $\beta_{e}$ and $\kappa$ was conducted using the least_squares function in the SCIPY library's optimization package, which implements a trustregion-reflexive algorithm [79]. Confidence intervals of $95 \%$ were computed using the nlparci function in MATLAB.

Relevant codes can be found at Ref. [80].

\section{APPENDIX C: AN ALTERNATIVE DERIVATION OF THE SNAIL-TRAIL CORRECTIVE FACTOR}

In this Appendix, we demonstrate that it is possible to derive Eq. (14), our expression for $\kappa(x, y)$, using a procedure given by Travasso et al. [70] and Vilanova et al. [71]. In those articles, the modeling framework is not a snail-trail system given by Eqs. (3) and (4), but instead a hybrid ABM that utilizes a discrete model for tip cell movement and a continuous model for capillary density. Despite such differences, this hybrid ABM contains a nondimensional value that couples the discrete and continuum models and is very similar to Eq. (14). By deriving our formula for $\kappa(x, y)$ using the logic outlined in Refs. [70,71], we aim to show that the two factors are equivalent and, hence, that both terms arise from a common physical mechanism. 
In Refs. [70,71], the coupling factor is calculated as "the ratio of the [vessel] material produced within the tip cell to the [area] of material swept as the cell migrates" [71]. In other words, the coupling factor in the hybrid ABM is given as

$$
\text { factor }=\frac{\text { area of vessel produced within time } \Delta t}{\text { area swept out by tip cells within time } \Delta t}=\frac{A_{1}}{A_{2}} .
$$

We may physically interpret Eq. (C1) as the tip cell "smearing" vessel density along the tunnel it creates within a given time step. In other words, the coupling factor arises as a result of the snail-trail assumption that new vessels proliferate along the path of moving tip cells. We remark that Eq. (14), our formula for $\kappa(x, y)$, also arises from this physical assumption.

We now calculate $\kappa(x, y)$ using Eq. (C1) and the discrete model described in Sec. III. We recall that new vessel production in the discrete model is equal to the number of times that tip cells move from a lattice site. Since tip cells move with probability $P_{m}$ in time $\Delta t$ and the area of a tip cell is given by $h^{2}$, the total new vessel area produced by a tip cell, on average, is equal to $A_{1}=h^{2} P_{m}$.
The area per lattice length that a tip cell sweeps out when it moves is also equal to $h^{2} P_{m}$. To calculate the total area swept out by the cell, however, this quantity must also be multiplied by the average distance that the tip cell travels. Following our logic from Sec. III, this latter value can be calculated as the expected net distance that a tip cell makes within a single jump, which is given by

$$
\begin{aligned}
\sqrt{\mathbb{E}\left[X_{\text {net }}\right]^{2}+\mathbb{E}\left[Y_{\text {net }}\right]^{2}} & =\sqrt{\left(P_{x^{+}}-P_{x^{-}}\right)^{2}+\left(P_{y^{+}}-P_{y^{-}}\right)^{2}} \\
& =\frac{1}{2} \sqrt{\left(g_{x}\right)^{2}+\left(g_{y}\right)^{2}},
\end{aligned}
$$

where $P_{x^{ \pm}}, P_{y^{ \pm}}, g_{x}$, and $g_{y}$ are the same values as in Eqs. (7) and (8). By applying Taylor's theorem and neglecting terms of $O\left(h^{3}\right)$ or smaller, we find that the expected distance that a single tip cell travels is approximately $k h\|\nabla c\|_{2}$, hence the total area swept out by the tip cell within time $\Delta t$ is approximately $A_{2}=h^{3} k P_{m}\|\nabla c\|_{2}$.

It is straightforward to derive Eq. (14) after substituting the above expressions for $A_{1}$ and $A_{2}$ into Eq. (C1) and defining $\mu:=P_{m} / \Delta t$ and $\chi:=P_{m} k h^{2} / \Delta t$.
[1] J. Li, T. Kim, and D. B. Szymanski, Dev. Biol. 451, 40 (2019).

[2] H. Motte and T. Beeckman, J. Exp. Bot. 70, 785 (2019).

[3] D. Goodwin and C. M. Nelson, Development 147, dev184499 (2020).

[4] V. D. Varner and C. M. Nelson, Semin. Cell Dev. Biol. 67, 170 (2017).

[5] D. Menshykau, O. Michos, C. Lang, L. Conrad, A. P. McMahon, and D. Iber, Nat. Commun. 10, 1 (2019).

[6] F. Costantini and R. Kopan, Dev. Cell 18, 698 (2010).

[7] C. L. Duran, D. W. Howell, J. M. Dave, R. L. Smith, M. E. Torrie, J. J. Essner, and K. J. Bayless, Compr. Physiol. 8, 153 (2017).

[8] A. Ochoa-Espinosa and M. Affolter, Cold Spring Harb. Perspect. Biol. 4, a008243 (2012).

[9] M. Potente, H. Gerhardt, and P. Carmeliet, Cell 146, 873 (2011).

[10] S. Barrientos, O. Stojadinovic, M. S. Golinko, H. Brem, and M. Tomic-Canic, Wound Repair Regen. 16, 585 (2008).

[11] W. Risau, Nature 386, 671 (1997).

[12] E. Hannezo and B. D. Simons, Curr. Opin. Cell Biol. 60, 99 (2019).

[13] A. Martin, M. R. Komada, and D. C. Sane, Med. Res. Rev. 23, 117 (2003).

[14] Y. M. Paulus and A. Sodhi, Handb. Exp. Pharmacol. 242, 271 (2017).

[15] N. Sawada and Z. Arany, Physiology 32, 290 (2017).

[16] F. M. Davis, A. Kimball, A. Boniakowski, and K. Gallagher, Curr. Diabetes Rep. 18, 1 (2018).

[17] C. Viallard and B. Larrivée, Angiogenesis 20, 409 (2017).

[18] H. M. Byrne, Nat. Rev. Cancer. 10, 221 (2010).

[19] L. Yadav, N. Puri, V. Rastogi, P. Satpute, and V. Sharma, J. Clin. Diagn. Res. 9, XE01 (2015).

[20] J. C. Bowersox and N. Sorgente, Cancer Res. 42, 2547 (1982).

[21] P. Carmeliet and R. K. Jain, Nature 473, 298 (2011).
[22] A. Szymborska and H. Gerhardt, Cold Spring Harb. Perspect. Biol. 10, a029223 (2018).

[23] C. Betz, A. Lenard, H. G. Belting, and M. Affolter, Development 143, 2249 (2016).

[24] L. K. Phng and H. Gerhardt, Dev. Cell 16, 196 (2009).

[25] M. L. Iruela-Arispe and G. E. Davis, Dev. Cell 16, 222 (2009).

[26] N. V. Mantzaris, S. Webb, and H. G. Othmer, J. Math. Biol. 49, 111 (2004).

[27] M. Scianna, C. G. Bell, and L. Preziosi, J. Theor. Biol. 333, 174 (2013).

[28] T. A. M. Heck, M.-M. Vaeyens, and H. Van Oosterwyck, Math. Model. Nat. Phenom. 10, 108 (2014).

[29] J. A. Flegg, S. N. Menon, H. M. Byrne, and D. L. S. McElwain, Bull. Math. Biol. 82, 1 (2020).

[30] K. A. Rejniak and A. R. A. Anderson, Wiley Interdiscip. Rev. Syst. Biol. Med. 3, 115 (2011).

[31] A. R. A. Anderson and M. A. J. Chaplain, Bull. Math. Biol. 60, 857 (1998).

[32] S. Pillay, H. M. Byrne, and P. K. Maini, Phys. Rev. E 95, 012410 (2017).

[33] T. Jackson and X. Zheng, Bull. Math. Biol. 72, 830 (2010).

[34] A. A. Qutub and A. S. Popel, BMC Syst. Biol. 3, 13 (2009).

[35] F. Spill, P. Guerrero, T. Alarcon, P. K. Maini, and H. M. Byrne, J. Math. Biol. 70, 485 (2015).

[36] M. Scianna, L. Munaron, and L. Preziosi, Prog. Biophys. Mol. Biol. 106, 450 (2011).

[37] A. L. Bauer, T. L. Jackson, and Y. Jiang, Biophys. J. 92, 3105 (2007).

[38] S. E. M. Boas, Y. Jiang, R. M. H. Merks, S. A. Prokopiou, and E. G. Rens, in Probabilistic Cellular Automata, edited by P.-Y. Louis and F. R. Nardi (Springer International Publishing, New York, 2018), Chap. 18, pp. 279-310.

[39] H. Perfahl, B. D. Hughes, T. Alarcón, P. K. Maini, M. C. Lloyd, M. Reuss, and H. M. Byrne, J. Theor. Biol. 414, 254 (2017). 
[40] L. L. Bonilla, V. Capasso, M. Alvaro, M. Carretero, and F. Terragni, Math. Biosci. Eng. 14, 45 (2017).

[41] L. L. Bonilla, V. Capasso, M. Alvaro, and M. Carretero, Phys. Rev. E 90, 062716 (2014).

[42] C. L. Stokes, D. A. Lauffenburger, and S. K. Williams, J. Cell Sci. 99, 419 (1991).

[43] J. A. Grogan, A. J. Connor, B. Markelc, R. J. Muschel, P. K. Maini, H. M. Byrne, and J. M. Pitt-Francis, Biophys. J. 112, 1767 (2017).

[44] M. H. Swat, S. D. Hester, A. Balter, R. W. Heiland, B. L. Zaitlen, and J. A. Glazier, in Systems Biology, edited by I. V. Maly (Humana Press, New York, 2009), Chap. 13, pp. 361-428.

[45] J. A. Flegg, S. N. Menon, P. K. Maini, and D. L. S. McElwain, Front. Physiol. 6, 00262 (2015).

[46] A. J. Connor, R. P. Nowak, E. Lorenzon, M. Thomas, F. Herting, S. Hoert, T. Quaiser, E. Shochat, J. Pitt-Francis, J. Cooper, P. K. Maini, and H. M. Byrne, J. R. Soc., Interface 12, 20150546 (2015).

[47] H. M. Byrne and M. A. J. Chaplain, Bull. Math. Biol. 57, 461 (1995).

[48] D. Balding and D. L. S. McElwain, J. Theor. Biol. 114, 53 (1985).

[49] S. Pillay, H. M. Byrne, and P. K. Maini, J. Math. Biol. 77, 1721 (2018).

[50] L. Edelstein, J. Theor. Biol. 98, 679 (1982).

[51] M. Jabed, P. M. J. Trevelyan, and G. P. Boswell, J. Theor. Biol. 438, 9 (2018).

[52] M. E. Orme and M. A. J. Chaplain, IMA J. Math. Med. Biol. 13, 73 (1996).

[53] P. Bastian, A. Chavarría-Krauser, C. Engwer, W. Jäger, S. Marnach, and M. Ptashnyk, J. Theor. Biol. 254, 99 (2008).

[54] M. E. Orme and M. A. J. Chaplain, IMA J. Math. Med. Biol. 14, 189 (1997).

[55] G. Pettet, M. A. J. Chaplain, D. L. S. McElwain, and H. M. Byrne, Proc. R. Soc. B 263, 1487 (1996).

[56] G. J. Pettet, H. M. Byrne, D. L. S. McElwain, and J. Norbury, Math. Biosci. 136, 35 (1996).

[57] L. Tweedy, P. A. Thomason, P. I. Paschke, K. Martin, L. M. Machesky, M. Zagnoni, and R. H. Insall, Science 369, eaay9792 (2020).

[58] K. Bentley, G. Mariggi, H. Gerhardt, and P. A. Bates, PLoS Comput. Biol. 5, e1000549 (2009).

[59] M. A. J. Chaplain, T. Lorenzi, and F. R. Macfarlane, J. Math. Biol. 80, 343 (2020).

[60] T. Hillen and K. J. Painter, J. Math. Biol. 58, 183 (2009).

[61] C. Ruhrberg, H. Gerhardt, M. Golding, R. Watson, S. Ioannidou, H. Fujisawa, C. Betsholtz, and D. T. Shima, Genes Dev. 16, 2684 (2002).
[62] H. Gerhardt, M. Golding, M. Fruttiger, C. Ruhrberg, A. Lundkvist, A. Abramsson, M. Jeltsch, C. Mitchell, K. Alitalo, D. Shima, and C. Betsholtz, J. Cell Biol. 161, 1163 (2003).

[63] A. N. Gordon-Weeks, S. Y. Lim, A. E. Yuzhalin, K. Jones, B. Markelc, K. J. Kim, J. N. Buzzelli, E. Fokas, Y. Cao, S. Smart, and R. Muschel, Hepatology 65, 1920 (2017).

[64] A. Uccelli, T. Wolff, P. Valente, N. Di Maggio, M. Pellegrino, L. Gürke, A. Banfi, and R. Gianni-Barrera, Swiss Med. Wkly. 149, w20011 (2019).

[65] V. R. Muthukkaruppan, L. Kubai, and R. Auerbach, J. Natl. Cancer Inst. 69, 699 (1982).

[66] M. J. Simpson, K. A. Landman, and B. D. Hughes, Physica A 388, 399 (2009).

[67] D. C. Markham, M. J. Simpson, and R. E. Baker, Phys. Rev. E 87, 062702 (2013).

[68] See Supplemental Material at http://link.aps.org/supplemental/ 10.1103/PhysRevE.102.062417 for supplementary figures referenced in the main text of this article.

[69] P. H. Wu, A. Giri, S. X. Sun, and D. Wirtz, Proc. Natl. Acad. Sci. U.S.A. 111, 3949 (2014).

[70] R. D. M. Travasso, E. C. Poiré, M. Castro, J. C. RodrguezManzaneque, and A. Hernández-Machado, PLoS ONE 6, e19989 (2011).

[71] G. Vilanova, I. Colominas, and H. Gomez, Comput. Mech. 53, 449 (2014).

[72] L. L. Bonilla, M. Carretero, and F. Terragni, Phys. Rev. E 94, 062415 (2016).

[73] C. J. Penington, B. D. Hughes, and K. A. Landman, Phys. Rev. E 84, 041120 (2011).

[74] C. J. Penington, B. D. Hughes, and K. A. Landman, Phys. Rev. E 89, 032714 (2014).

[75] K. Bentley, H. Gerhardt, and P. A. Bates, J. Theor. Biol. 250, 25 (2008).

[76] M. S. Alnaes, J. Blechta, J. Hake, A. Johansson, B. Kehlet, A. Logg, C. Richardson, J. Ring, M. E. Rognes, and G. N. Wells, Arch. Numer. Softw. 3, 9 (2015).

[77] A. Logg and G. N. Wells, ACM Trans. Math. Softw. 37, 1 (2020).

[78] A. Logg, G. N. Wells, and J. Hake, DOLFIN: a C++/Python finite element library, in Automated Solution of Differential Equations by the Finite Element Method, edited by A. Logg, K.-A. Mardal, and G. N. Wells, Lecture Notes in Computational Science and Engineering Vol. 84, (Springer, Berlin, 2012), pp. 173-225.

[79] T. F. Coleman and Y. Li, SIAM J. Optim. 6, 1040 (1996).

[80] https://github.com/wdmartinson/snail-trail-Pillay-angiogene sis-models. 